\title{
A GENERAL FRAMEWORK OF LOW REGULARITY INTEGRATORS
}

\author{
FRÉDÉRIC ROUSSET AND KATHARINA SCHRATZ
}

\begin{abstract}
We introduce a new general framework for the approximation of evolution equations at low regularity and develop a new class of schemes for a wide range of equations under lower regularity assumptions than classical methods require. In contrast to previous works, our new framework allows a unified practical formulation and the construction of the new schemes does not rely on any Fourier based expansions. This allows us for the first time to overcome the severe restriction to periodic boundary conditions, to embed in the same framework parabolic and dispersive equations and to handle nonlinearities that are not polynomial. In particular, as our new formalism does no longer require periodicity of the problem, one may couple the new time discretisation technique not only with spectral methods, but rather with various spatial discretisations. We apply our general theory to the time discretization of various concrete PDEs, such as the nonlinear heat equation, the nonlinear Schrödinger equation, the complex Ginzburg-Landau equation, the half wave and Klein-Gordon equations, set in $\Omega \subset \mathbb{R}^{d}, d \leq 3$ with suitable boundary conditions.
\end{abstract}

\section{INTRODUCTION}

We consider a general class of evolution equations under the form

$$
\partial_{t} u-\mathcal{L} u=f(u, \bar{u}) \quad(t, x) \in \mathbb{R} \times \Omega
$$

with $\Omega \subset \mathbb{R}^{d}$. We add an initial condition

$$
u_{/ t=0}=u_{0}
$$

and when $\partial \Omega \neq \emptyset$ some appropriate homogeneous boundary conditions. The unknown $u$ can be complex valued or real-valued. The precise assumptions for the general problem (1) will be given in Section 1.2 and concrete examples that fit the assumptions are illustrated in Section 1.3.

In the last decades, a large variety of discretisation techniques was introduced for the time resolution of evolution equations of type (1) reaching from splitting methods over exponential integrators up to Runge-Kutta and Lawson schemes $[8,9,10,11,12,13,16,20,26]$. While such classical discretisation techniques in general provide a good approximation to smooth solutions of partial differential equations (PDEs), they often drastically break down at low regularity: rough data and high oscillations can cause severe order reduction leading to loss of convergence and huge computational costs.

Lack of smoothness is thereby mostly negligible for parabolic problems: thanks to the parabolic smoothing property the solution is regularised away from time $t=0$ such that classical schemes in general provide a good approximation as time evolves. This stands in stark contrast to the dispersive setting where no pointwise smoothing can be expected. Rough (or oscillatory) data spreads in time and in space which causes the breakdown of classical approximation techniques. The control of nonlinear terms in dispersive PDEs at low regularity is thus an ongoing challenge in computational mathematics at large.

Recently, a new type of discretisation (so-called resonance based discretisation) was introduced for various dispersive equations with periodic boundary conditions (that is $\Omega=\mathbb{T}^{d}$ ) when the linear part is defined by a differential operators $\mathcal{L}=\sum_{i=1}^{d} \partial_{x_{i}}^{n}$ with $n \in \mathbb{N}$. First for the periodic Korteweg-de Vries equation ([14]), then for periodic Schrödinger equations ([22]) and lately for other periodic dispersive equations such as Boussinesq ([25]) and Dirac equations ([27]). The 
main idea behind the new discretisation technique is the following: Instead of discretising the evolution equation (1) directly with classical techniques based on a Taylor series expansion of the exact solution, one embeds the underlying structure of nonlinear frequency interactions into the numerical discretisation. The latter is achieved by expanding the exact solution into a Fourier series expansion $u(t, x)=\sum_{k \in \mathbb{Z}^{d}} \hat{u}_{k}(t) e^{i k x}$. In the discretisation of the equation written on the Fourier side one can then (easily) tackle the nonlinear interactions of the Fourier modes and embed their dominant parts into the numerical discretisation. In many cases this enables us to solve the dispersive PDE under (much) lower regularity assumptions than required by classical numerical schemes which are based on linearised frequency approximations. For an extensive overview on the comparison of this approach to classical methods, we refer to [5].

While the novel approach allowed us to solve dispersive PDEs in a more general setting, i.e., under lower regularity assumptions, its main drawback lies in the fact that each and every equation has to be carefully analyzed on its own since the nonlinear frequency interaction strongly differs from PDE to PDE. With the aid of decorated tree series we could recently show that a general high-order framework of resonance based discretisations does exist [5]. However, in order to obtain a practical implementation each scheme has to be derived separately through involved ButcherConnes-Kreimer Hopf algebras and their corresponding co-products.

Consequently, while resonance based approaches allow us to solve dispersive PDEs under (much) lower regularity assumptions than classical schemes require, they face three main obstacles:

(A) They are restricted to periodic boundary conditions due to their strong dependence on the Fourier series expansion of the exact solution.

(B) They are limited to classical differential operators of type $\mathcal{L}=\sum_{i=1}^{d} \partial_{x_{i}}^{n}$ with $n \in \mathbb{N}$, e.g.,

$$
\mathcal{L}=-\Delta, \partial_{x}, \partial_{x}^{3}, \ldots,
$$

in order to involve only polynomials in the frequencies in the interaction phase and to allow the dominant parts to be straightforwardly extracted. The latter, however, excludes important classes of equations, such as Klein-Gordon, or wave type systems which does not involve polynomials, but rather interactions of square roots of frequencies (for example for quadratic nonlinearities $\sqrt{(j+k)^{2}+m^{2}}-\sqrt{j^{2}+m^{2}}-\sqrt{k^{2}+m^{2}},|j+k|-|j|-|k|$, etc).

(C) The lack of a unified practical formulation of the schemes which is the main advantage of Runge-Kutta methods, splitting methods, exponential integrators and other classical discretisation techniques.

1.1. Aim of the paper. In this work we present a novel framework of low regularity integrators which allows us to overcome the above obstacles. The central idea lies in embedding the underlying oscillatory structure of (1) into our numerical discretisation (without employing any Fourier based techniques). This is achieved by introducing filtered oscillations (cf. (39)) which allow us to treat the dominant oscillations, triggered by the operator $-\mathcal{L}+\overline{\mathcal{L}}$ exactly, while only approximating the lower order parts by a stabilised Taylor series expansion. This framework yields a new class of schemes that can be used on a wide range of equations under lower regularity assumptions than classical methods require. The new framework allows for a unified practical formulation (C) while still embedding the central oscillations in the discretisation. The construction of the new schemes does not rely on any Fourier based expansions. This will allow us to solve a large class of equations at low regularity without requiring periodicity of the problem (A). In particular, as the spatial domain is no longer restricted to periodicity, one may couple the new time discretisation technique not only with spectral methods, but rather with various spatial discretisations (e.g., finite differences, finite element methods). The practical implementation on general domains will require, as for classical splitting and exponential integrators methods, suitable Krylov space methods in order to approximate the exponential $e^{t \mathcal{L}}$, respectively, the action of the $\varphi-$ functions. A fully 
discrete analysis with various spatial discretisation methods is plan of future research. In addition of overcoming periodicity, the new general framework also allows us for the first time to deal in the same formulation with parabolic and dispersive or hyperbolic problems (B). The latter will open up this new low regularity time discretisation framework to a larger class of equations including for instance heat equations, reaction-diffusion problems and wave-type systems. Furthermore, in contrast to previous works, our framework is no longer restricted to strict polynomial nonlinearities and we can treat for instance the Sine-Gordon equation with our new framework. We carry out an overarching abstract convergence analysis for our new class of schemes in the general setting (1). The abstract error bounds we establish apply to a lot of examples. We illustrate them for nonlinear heat, Schrödinger and Ginzburg-Landau type equations as well as the half wave, Klein-Gordon and wave equations.

The new first- and second-order schemes together with their main convergence result are presented in Section 2. In Section 3 and 4 we carry out an expansion of the underlying oscillations up to first- and second-order, respectively. These expansions together with their improved local error structure motivate the new numerical schemes. We focus on first- and second-order methods. However, our framework can be extended to higher order. The error analysis at first- and second-order is presented in Section 3.2 and 4.1, respectively. Finally, in Section 5 we illustrate our convergence analysis on various examples.

1.2. Assumptions. The linear operator $\mathcal{L}$ is defined on a Hilbert space $X$ of complex valued functions $u \in \mathbb{C}$ with norm denoted by $\|\cdot\|$ and domain $\mathcal{D}(\mathcal{L})$. We assume that the complex conjugation $u \mapsto \bar{u}$ is an isometry on $X$. When $\partial \Omega \neq 0$, the boundary conditions will be encoded in the choice of the domain of the operator $\mathcal{L}$. We shall also use the operator $\overline{\mathcal{L}}$ defined by $\overline{\mathcal{L}} u=\overline{\mathcal{L}} \bar{u}$. In the following we assume that the nonlinearity $f$ is tensorized under the form

$$
f(v, w)=\mathcal{B}(F(v) \cdot G(w)), \quad F, G: \mathbb{C} \rightarrow \mathbb{C}^{J},
$$

where we use the notation $X \cdot Y=\sum_{k} X_{k} Y_{k}, \quad X, Y \in \mathbb{C}^{J}$, and $\mathcal{B}$ is a linear operator.

Assumption 1.1. Our main assumptions on $\mathcal{L}$ are the following

i) $\mathcal{L}$ generates a strongly continuous semigroup $\left\{e^{t \mathcal{L}}\right\}_{t \geq 0}$ of contractions on $X$;

ii) $\mathcal{A}=-\mathcal{L}+\overline{\mathcal{L}}$ generates a group $\left\{e^{t \mathcal{A}}\right\}_{t \in \mathbb{R}}$ of unitary operators on $\mathrm{X}$;

iii) $\mathcal{L}$ and $\overline{\mathcal{L}}$ commute: $[\mathcal{L}, \overline{\mathcal{L}}]=0$.

The above assumptions allow us to deal in an unified framework with parabolic, dispersive as well as mixed equations, we shall give examples in Section 1.3 below. Note that the second assumption is automatically verified if $\mathcal{A}=0$ so in particular when $\mathcal{L}$ is real for real functions.

The consequences of the above assumptions that we shall use are the following. Since $\left\{e^{t \mathcal{L}}\right\}_{t \geq 0}$ is a strongly continuous semigroup of contractions it holds that

$$
\left\|e^{t \mathcal{L}}\right\|_{\mathcal{L}(X)} \leq 1
$$

We can define powers of $\mathcal{L}, \mathcal{L}^{\alpha}$ for $\alpha \in \mathbb{R}$ and we shall denote the domain $\mathcal{D}\left(\mathcal{L}^{\alpha}\right)$ by $X^{\alpha}$ (with the convention that $X^{0}=X$ ), the graph norm will be denoted for $\alpha \geq 0$ by

$$
\|u\|_{\alpha}=\|u\|+\left\|\mathcal{L}^{\alpha} u\right\| .
$$

We could also deal with general strongly continuous semigroups which then satisfies

$$
\left\|e^{t \mathcal{L}} u\right\| \leq M e^{\omega t}
$$

for some $\omega \geq 0$ but this is not needed for the examples that we shall study. 
Assumption 1.2. To handle the "nonlinearity" $f$, we assume that there exists $a_{1}>a_{0} \geq 0$ such that for every $\alpha \in\left[a_{0}, a_{1}\right]$,

$$
\|f(u, \bar{u})\|_{\alpha} \leq C_{\alpha}\left(\|u\|_{a_{0}}\right)\|u\|_{\alpha}, \quad\|f(u, \bar{u})-f(v, \bar{v})\|_{a_{0}} \leq C_{a_{0}}\left(\|u\|_{a_{0}},\|v\|_{a_{0}}\right)\|u-v\|_{a_{0}}
$$

where $C_{\alpha}(\cdot)$ denotes a continuous non-decreasing functions of its arguments.

In the above assumption, $a_{0}$ is the minimal regularity needed to have a standard well-posedness result, while $a_{1}$ stands for the maximal regularity that we can propagate without imposing further compatibility conditions. The threshold $a_{1}$ will be meaningful only when we consider domains with boundaries, on the whole space or the torus $a_{1}$ can be arbitrarily large.

1.3. Examples. The main examples which will be covered by the above framework are

- Nonlinear heat equations

$$
\partial_{t} u-\Delta u=f(u, \bar{u}), \quad \text { i.e., } \quad \mathcal{L}=\Delta, \mathcal{A}=0,
$$

where $f$ is sufficiently smooth

- Nonlinear Schrödinger equations

$$
i \partial_{t} u+\Delta u= \pm|u|^{2 m} u, m \in \mathbb{N} \quad \text { i.e., } \quad \mathcal{L}=i \Delta, \mathcal{A}=-2 i \Delta, \quad f(u, \bar{u})= \pm i u^{m+1} \bar{u}^{m} .
$$

- Complex Ginzburg Landau equations

$$
\begin{aligned}
& \partial_{t} u-\alpha \Delta u=\gamma u\left(1-|u|^{2}\right), \alpha, \gamma \in \mathbb{C}, \operatorname{Re} \alpha \geq 0, \\
& \text { i.e., } \quad \mathcal{L}=\alpha \Delta, \mathcal{A}=-2 i \operatorname{Im} \alpha \Delta, f(u, \bar{u})=\gamma u(1-u \bar{u})
\end{aligned}
$$

- Half wave equation

$$
i \partial_{t} u+|\nabla| u= \pm|u|^{2} u, \quad \text { i.e., } \quad \mathcal{L}=i|\nabla|, \quad f(u, \bar{u})= \pm u^{2} \bar{u}
$$

- Klein-Gordon and wave-type equations reformulated as first-order systems

$$
\partial_{t t} u-\Delta u+m^{2} u=f(u)
$$

with $f$ smooth enough, for example, $f(u)=u^{p}$ or Sine-Gordon equations $f(u)=\sin (u)$.

We shall apply our general theory to the time discretization of the above partial differential equations set in $\Omega \subset \mathbb{R}^{d}, d \leq 3$ with suitable boundary conditions. This will be the aim of the last part of the paper, see Section 5.

\section{MAIN RESUlts}

We shall deal with mild solutions of (1), (2) which satisfy the Duhamel formula

$$
u(t)=e^{t \mathcal{L}} u_{0}+\int_{0}^{t} e^{(t-\xi) \mathcal{L}} f(u(\xi), \bar{u}(\xi)) d \xi .
$$

By using Assumptions 1.1, 1.2, we can easily get from a Banach fixed point argument the following local existence result:

Theorem 1. Under the Assumptions 1.1, 1.2, for every $u_{0} \in X^{a_{0}}$, there exists $T>0$ and a unique solution $u \in \mathcal{C}\left([0, T], X^{a_{0}}\right)$ solution of (5). Moreover if $u_{0} \in X^{\alpha}, \alpha \in\left(a_{0}, a_{1}\right]$, then we also have $u \in \mathcal{C}\left([0, T], X^{\alpha}\right)$.

The aim of this paper is to introduce low-regularity integrators for the approximation of Duhamel's formula (5). We shall call these schemes Duhamel integrators. 
2.1. First-order Duhamel integrator. At first-order our new Duhamel integrator takes the form for $l \geq 0$

$$
u^{\ell+1}=\Phi_{\text {num }, 1}^{\tau}\left(u^{\ell}\right)=e^{\tau \mathcal{L}}\left(u^{\ell}+\tau \mathcal{B}\left(F\left(u^{\ell}\right) \cdot \varphi_{1}(\tau \mathcal{A}) G\left(\overline{u^{\ell}}\right)\right)\right) \quad \text { with } \quad \varphi_{1}(z)=\frac{e^{z}-1}{z}
$$

and we set $u^{0}=u_{0}$. Let us recall that we have set $\mathcal{A}=-\mathcal{L}+\overline{\mathcal{L}}$. Thanks to Assumption (1.1), Stone's Theorem implies that $\mathcal{A}$ is under the form $i \mathcal{M}$ with $\mathcal{M}$ self-adjoint. We can therefore define $\varphi_{1}(\tau \mathcal{A})$ by using the functional calculus for self-adjoint operator, this yields a bounded operator since $\varphi_{1}$ is bounded on $i \mathbb{R}$.

It will be convenient to set

$$
\Phi_{\text {num }, 1}^{\tau}\left(u^{\ell}\right)=e^{\tau \mathcal{L}}\left(u^{\ell}+\tau \mathcal{B}\left(F\left(u^{\ell}\right) \cdot \varphi_{1}(\tau \mathcal{A}) G\left(\overline{u^{\ell}}\right)\right)\right)=e^{\tau \mathcal{L}}\left(u^{\ell}+\tau \Psi_{\text {num }, 1}^{\tau}\left(u^{l}\right)\right) .
$$

Let us define for a function $H\left(v_{1}, \cdots v_{n}\right), n \geq 1$ and a linear operator $L$ the commutator type term $\mathcal{C}[H, L]$ by

$$
\mathcal{C}[H, L]\left(v_{1}, \cdots, v_{n}\right)=-L\left(H\left(v_{1}, \cdots v_{n}\right)\right)+\sum_{i=1}^{n} D_{i} H\left(v_{1}, \cdots, v_{n}\right) \cdot L v_{i}
$$

where $D_{i} H$ stands for the partial differential of $H$ with respect to the variable $v_{i}$. The estimate of this type of terms will be crucial in order to estimate the local error of our schemes. From Assumption 1.2, if $a_{1}$ can be taken bigger than 1, we can get that

$$
\|\mathcal{C}[f, \mathcal{L}](v, w)\| \leq C\left(\|v\|_{a_{0}},\|w\|_{a_{0}}\right)\left(\|v\|_{1}+\|w\|_{1}\right) .
$$

Nervertheless, this estimate is very crude since each term in the expression of $\mathcal{C}[f, \mathcal{L}]$ actually satisfies the above estimate. By using this estimate in the analysis of our first-order scheme we would get first-order convergence in $X$ for data in $X^{1}$ without any improvement compared to classical schemes. However, if $\mathcal{L}$ is a differential operator of order $m$, we can get a better estimate by using the Leibnitz formula which implies that $\mathcal{C}[f, \mathcal{L}]$ actually involves at most only $m-1$ derivatives of $u$ so that a better estimate can be expected. If $\mathcal{L}$ is not a differential operator but satisfies a generalized Leibnitz rule, such an improvement can also be obtained as we will see on the examples.

We shall now state an abstract assumption about the estimate of these commutator terms which will fix the needed regularity for the convergence of the new scheme (6). In order to get a result as general as possible, we take some $\alpha_{0} \in\left[0, a_{1}\right]$ and we will measure errors in $X^{\alpha_{0}}$.

Assumption 2.1. There exists $\alpha_{1} \in\left(a_{0}, a_{1}\right]$ such that for every $v, w \in X^{\alpha_{1}}$,

$$
\begin{aligned}
& \|\mathcal{C}[f, \mathcal{L}](v, w)\|_{\alpha_{0}} \leq C_{\alpha_{0}}\left(\|v\|_{\alpha_{1}},\|w\|_{\alpha_{1}}\right), \\
& \left\|\mathcal{B}\left(F(v) \cdot e^{s \mathcal{A}} \mathcal{C}[G, \mathcal{A}](w)\right)\right\|_{\alpha_{0}} \leq C_{\alpha_{0}}\left(\|v\|_{\alpha_{1}},\|w\|_{\alpha_{1}}\right), \quad \forall s \in \mathbb{R} .
\end{aligned}
$$

The second estimate is uniform with respect to $s$.

In the above assumption $a_{1}$ stands for the minimal regularity needed to estimate the commutator terms (and thus the local error as we shall see) in the space that we have chosen to measure the error which is $X^{\alpha_{0}}$.

Note that if we choose to measure errors in $X$ so that $\alpha_{0}=0$, then if we can check on a concrete example that $\alpha_{1}<1$ we obtain indeed an improvement on the trivial estimate (9). Due to the favorable local error structure of our scheme which involves these commutator type terms, this will allow us to get first-order convergence in $X$ for data in $X^{\alpha_{1}}$ instead of $X^{1}$. 
To make our point more concrete let us consider the simple case $\mathcal{L}=\Delta$ on the torus $\mathbb{T}^{d}$, and $f(u)=u^{2}$. Note that $X^{\alpha}=H^{2 \alpha}$ in this case. We have the explicit formula

$$
\mathcal{C}[f, \mathcal{L}](v, w)=-2 \sum_{k=1}^{d} \partial_{k} v \partial_{k} w .
$$

If we choose to measure the error in $L^{2}$, so that $\alpha_{0}=0$, we need $\partial_{k} v \partial_{k} w \in L^{2}$. If $d \leq 4$, we can take $v, w \in W^{1,4}$ and thus, since $H^{\frac{d}{4}} \subset L^{4}$, this yields $v w \in H^{1+\frac{d}{4}}$. This means that we can take $a_{1}=\frac{1}{2}+\frac{d}{8}$ which is strictly smaller than 1 for $d \leq 3$.

Before stating our main result for first-order convergence, our last technical Assumption is the following

Assumption 2.2. Let $\alpha_{0}, \alpha_{1}$ be given as above, we assume that

$$
\|f(u, \bar{u})-f(v, \bar{v})\|_{\alpha_{0}} \leq C_{\alpha_{0}}\left(\|u\|_{a_{0}},\|v\|_{a_{0}}\right)\|u-v\|_{\alpha_{0}}, \quad\|f(u, \bar{u})\|_{\alpha_{0}} \leq C_{\alpha_{0}}\left(\|u\|_{\alpha_{1}}\right),
$$

and that the numerical flux is such that

$$
\begin{aligned}
& \left\|\Psi_{\text {num }, 1}^{\tau}(u)\right\|_{\alpha_{1}} \leq C_{\alpha_{1}}\left(\|u\|_{\alpha_{1}}\right) \\
& \left\|\Psi_{\text {num }, 1}^{\tau}(u)-\Psi_{\text {num }, 1}^{\tau}(v)\right\|_{\alpha_{0}} \leq C_{\alpha_{0}}\left(\|u\|_{a_{0}},\|v\|_{a_{0}}\right)\|u-v\|_{\alpha_{0}} \\
& \left\|\Psi_{\text {num }, 1}^{\tau}(u)-\Psi_{\text {num }, 1}^{\tau}(v)\right\|_{a_{0}} \leq C_{a_{0}}\left(\|u\|_{a_{0}},\|v\|_{a_{0}}\right)\|u-v\|_{a_{0}} .
\end{aligned}
$$

Note that we actually need both (14), (15) only when $\alpha_{0}<a_{0}$. This assumption will be used to prove boundedness for the numerical solution in $X^{a_{0}}$ and convergence in $X^{\alpha_{0}}$.

The global error estimate for the first-order Duhamel integrator (6) then takes the form.

Theorem 2. Let Assumptions 1.1, 1.2 hold, let us choose some $\alpha_{0} \in\left[0, a_{1}\right]$ such that Assumptions 2.1, 2.2 for some $\alpha_{1} \in\left(a_{0}, a_{1}\right]$ hold. Then, for every $u_{0} \in X^{\alpha_{1}}, \alpha_{1}$ given by Assumption 2.1, let $T>0$ and $u \in \mathcal{C}\left([0, T], X^{\alpha_{1}}\right)$ the unique solution of (5) given by Theorem 1 , let $u^{n}$ denote the numerical solution given by (6). Then there exists $C_{T}>0$ such that

$$
\left\|u(n \tau)-u^{n}\right\|_{\alpha_{0}} \leq C_{T} \tau, \quad 0 \leq n \tau \leq T .
$$

This abstract result will be applied to the concrete examples presented in Section 5. We will mainly have to check that Assumption 2.1 indeed holds true for some $\alpha_{1}<\alpha_{0}+1$ to get a concrete first-order convergence result that holds true for rougher data than classical schemes. One of the interest of this general theory is that different types of PDEs (parabolic, hyperbolic, dispersive) can be covered in the same unified framework. Nevertheless, the abstract proof will not use any fine structure of the PDE (for example smoothing effect for parabolic equations, Strichartz estimates in exterior domains for dispersive equations, etc). Using these specific properties when it is possible on a concrete example would allow to reduce again the smoothness of the initial data, see, e.g. $[11,23]$.

In certain cases we can relate our scheme (6) to more classical schemes.

Parabolic setting. In the parabolic setting $\mathcal{A}=0$ (or in case of nonlinearities depending only on $u$, i.e., $f(u, \bar{u})=f(u)$ ), the scheme (6) collapses to the classical exponential Euler method

$$
u^{\ell+1}=e^{\tau \mathcal{L}}\left(u^{\ell}+\tau f\left(u^{\ell}, \bar{u}^{\ell}\right)\right) .
$$

Filtered Lie splitting. For a nonlinearity (3) with $\mathcal{B}=1$ and polynomial $F(v)=v^{p}$ the low regularity scheme (6) can also be seen as a filtered Lie splitting scheme with filter function

$$
\Psi(\tau)=\varphi_{1}(\tau \mathcal{A})
$$

Indeed, the approximation

$$
\left.u^{\ell+1}=e^{\tau \mathcal{L}} \mathrm{e}^{\tau \frac{1}{p} F^{\prime}\left(u^{\ell}\right) \cdot\left(\Psi(\tau) G\left(\overline{u^{\ell}}\right)\right.}\right) u^{\ell}
$$


with filter function (17) introduces a similar error structure as the scheme (6). In the parabolic setting $\mathcal{A}=0$ the filter function (17) thereby naturally reduces to $\Psi(\tau) \equiv 1$ and the filtered Lie splitting (18) collapses to a classical Lie splitting. In the dispersive setting $\mathcal{L} \neq \overline{\mathcal{L}}$, on the other hand, the filtered splitting (18) allows for an improved error structure similar to the convergence result given in Theorem 2, see also Remark 22 for the example of the cubic Schrödinger equation. For details on filter functions we refer to [10] and the references therein.

2.2. Second-order Duhamel integrator. At second-order our new Duhamel integrator takes the form

$$
\begin{aligned}
u^{\ell+1} & =e^{\tau \mathcal{L}} u^{\ell}+\tau e^{\tau \mathcal{L}_{\mathcal{B}}}\left(F\left(u^{\ell}\right) \cdot \varphi_{1}(\tau \mathcal{A}) G\left(\bar{u}^{\ell}\right)\right)+\tau e^{\tau \mathcal{L}} \mathcal{B}\left(F\left(u^{\ell}\right) \cdot \varphi_{2}(\tau \mathcal{A}) \delta_{\tau}\left(e^{-\tau \mathcal{A}} G\left(e^{\tau \mathcal{A}} \bar{u}^{\ell}\right)\right)\right) \\
& +\tau \delta_{\xi}\left(e^{(\tau-\xi) \mathcal{L}} \mathcal{B}\left(F\left(e^{\xi \mathcal{L}} u^{\ell}\right) \cdot \varphi_{2}(\tau \mathcal{A}) G\left(e^{\xi \mathcal{L}} \bar{u}^{\ell}\right)\right)\right)_{/ \xi=\tau}+\frac{\tau^{2}}{2} e^{\tau \mathcal{L}}\left(D_{1} f^{\ell} \cdot f^{\ell}+D_{2} f^{\ell} \cdot \overline{f^{\ell}}\right) \\
& =\Phi_{\text {num }, 2}^{\tau}\left(u^{\ell}\right)=e^{\tau \mathcal{L}}\left(u^{\ell}+\tau \Psi_{\text {num }, 2}^{\tau}\left(u^{\ell}\right)\right)
\end{aligned}
$$

where $f^{\ell}=f\left(u^{\ell}, \bar{u}^{\ell}\right)$ and we use the notation of the standard shift operator

$$
\delta_{\tau} g(\tau)=g(\tau)-g(0) .
$$

Note that in general $D_{1} f\left(u^{\ell}, \bar{u}^{\ell}\right)$ and $D_{2} f\left(u^{\ell}, \bar{u}^{\ell}\right)$ can be calculated analytically. Nevertheless on concrete examples, they could also be approximated by standard finite differences. Similarly to the first-order scheme (6), its second-order counterpart (19) introduces an improved commutator-type error structure.

In order to analyze our second-order scheme, we need to introduce second-order commutators. For $H\left(v_{1}, \cdots v_{n}\right)$ and a linear operator $L$, we define the iterated "commutator"

$$
\mathcal{C}^{2}[H, L]\left(v_{1}, \cdots, v_{n}\right)=\mathcal{C}[\mathcal{C}[H, L], L]\left(v_{1}, \cdots, v_{n}\right) .
$$

We shall again measure the error in $X^{\alpha_{0}}$ for some given $\alpha_{0}$. The counterpart of Assumption 2.1 will be the following

Assumption 2.3. There exists $\alpha_{2} \in\left(a_{0}, a_{1}\right]$ such that for every $v, w \in X^{\alpha_{2}}$,

$$
\begin{aligned}
& \left\|\mathcal{C}^{2}[f, \mathcal{L}](v, w)\right\|_{\alpha_{0}} \leq C_{\alpha_{0}}\left(\|v\|_{\alpha_{2}},\|w\|_{\alpha_{2}}\right), \\
& \left\|\mathcal{B}\left(F(v) \cdot e^{s \mathcal{A}} \mathcal{C}^{2}[G, \mathcal{A}](w)\right)\right\|_{\alpha_{0}} \leq C_{\alpha_{0}}\left(\|v\|_{\alpha_{2}},\|w\|_{\alpha_{2}}\right), \quad \forall s \in \mathbb{R}, \\
& \left\|\mathcal{C}\left[\Psi_{s}, \mathcal{L}\right](v, w)\right\|_{\alpha_{0}} \leq C_{\alpha_{0}}\left(\|v\|_{\alpha_{2}},\|w\|_{\alpha_{2}}\right), \quad \forall s \in \mathbb{R},
\end{aligned}
$$

where $\Psi_{s}(v, w)=\mathcal{B}\left(F(v) \cdot e^{s \mathcal{A}} \mathcal{C}[G, \mathcal{A}](w)\right)$. The second and third estimates are uniform with respect to $s$.

Again in the case $\alpha_{0}=0$, a rough estimate that does not use the commutator structure would allow to estimate these commutators for $v, w$ in $X^{2}$ which would produce second-order convergence of the scheme in $X$ for data in $X^{2}$ like other classical schemes. If we can check on a concrete example by using the commutator structure that $\alpha_{2}<2$, then we can get improved second-order convergence of the scheme in $X$ for data only in $X^{\alpha_{2}}$.

The counterpart of Assumption 2.2 will be 
Assumption 2.4. Let $\alpha_{0}, \alpha_{2}$ be given as above, we assume that

$$
\begin{aligned}
& \|f(u, \bar{u})-f(v, \bar{v})\|_{\alpha_{0}} \leq C_{\alpha_{0}}\left(\|u\|_{a_{0}},\|v\|_{a_{0}}\right)\|u-v\|_{\alpha_{0}}, \\
& \left\|L^{k}(f(u, \bar{u}))\right\|_{\alpha_{0}}+\left\|D_{i} f(u, \bar{u}) \cdot L u\right\|_{\alpha_{0}}+\left\|D_{i} f(u, \bar{u}) \cdot g(v, \bar{v})\right\|_{\alpha_{0}} \leq C_{\alpha_{0}}\left(\|u\|_{\alpha_{2}},\|v\|_{\alpha_{2}}\right), \\
& \text { for } k=0,1, i=1,2, w \in\{\mathcal{L} u, \overline{\mathcal{L} u}, f(v, \bar{v}), \bar{f}(v, \bar{v})\}, \\
& \left\|L\left(D_{i} f(u, \bar{u}) \cdot g(v, \bar{v})\right)\right\|_{\alpha_{0}}+\left\|D_{i j}^{2} f(u, \bar{u}) \cdot\left(w_{1}, w_{2}\right)\right\|_{\alpha_{0}} \leq C_{\alpha_{0}}\left(\|u\|_{\alpha_{2}},\|v\|_{\alpha_{2}},\|w\|_{\alpha_{2}}\right), \\
& \text { for } i, j \in\{1,2\}, w_{i} \in\{\mathcal{L} w, \overline{\mathcal{L}} \bar{w}, f(v, v), \bar{f}(v, \bar{v})\},
\end{aligned}
$$

and for the numerical flux that

$$
\begin{aligned}
& \left\|\Psi_{\text {num }, 2}^{\tau}(u)\right\|_{\alpha_{2}} \leq C_{\alpha_{2}}\left(\|u\|_{\alpha_{2}}\right), \\
& \left\|\Psi_{\text {num }, 2}^{\tau}(u)-\Psi_{\text {num }, 2}^{\tau}(v)\right\|_{\alpha_{0}} \leq C_{a_{0}}\left(\|u\|_{a_{0}},\|v\|_{a_{0}}\right)\|u-v\|_{\alpha_{0}}, \\
& \left\|\Psi_{\text {num }, 2}^{\tau}(u)-\Psi_{\text {num }, 2}^{\tau}(v)\right\|_{a_{0}} \leq C_{\alpha_{0}}\left(\|u\|_{a_{0}},\|v\|_{a_{0}}\right)\|u-v\|_{a_{0}} .
\end{aligned}
$$

To check the above assumptions on concrete examples, we will be sometimes obliged to take $a_{0}$ bigger than for first-order convergence, nevertheless we did not change the notation. Our secondorder convergence result then reads:

Theorem 3. Let Assumptions 1.1, 1.2 hold, let us choose some $\alpha_{0} \in\left[0, a_{1}\right]$ such that Assumptions 2.3, 2.4 for some $\alpha_{2} \in\left(a_{0}, a_{1}\right]$ hold. Then, for every $u_{0} \in X^{\alpha_{2}}, \alpha_{2}$ given by Assumption 2.3, let $T>0$ and $u \in \mathcal{C}\left([0, T], X^{\alpha_{2}}\right)$ the unique solution of (5) given by Theorem 1 , let $u^{n}$ denote the numerical solution given by (19). Then there exists $C_{T}>0$ such that

$$
\left\|u(n \tau)-u^{n}\right\|_{\alpha_{0}} \leq C_{T} \tau^{2}, \quad 0 \leq n \tau \leq T .
$$

In the parabolic case we can again relate our new second-order scheme to classical methods.

Remark 4 (Parabolic case). Note that in the parabolic case $(\mathcal{A}=0)$ the second-order scheme (19) simplifies to an exponential Runge-Kutta method

$$
\begin{aligned}
u^{\ell+1} & =e^{\tau \mathcal{L}} u^{\ell}+\tau e^{\tau \mathcal{L}} f\left(u^{\ell}, \bar{u}^{\ell}\right)+\frac{\tau}{2} e^{\tau \mathcal{L}} \delta_{\tau} e^{-\tau \mathcal{L}} f\left(e^{\tau \mathcal{L}} u^{\ell}, e^{\tau \mathcal{L}} \bar{u}^{\ell}\right) \\
& +\frac{\tau^{2}}{2} e^{\tau \mathcal{L}}\left(D_{1} f\left(u^{\ell}, \bar{u}^{\ell}\right) \cdot f\left(u^{\ell}, \bar{u}^{\ell}\right)+D_{2} f\left(u^{\ell}, \bar{u}^{\ell}\right) \cdot \overline{f\left(u^{\ell}, \bar{u}^{\ell}\right)}\right) .
\end{aligned}
$$

Remark 5. The presented idea can be extended to higher order by carrying out higher order stabilised Taylor series expansions of the filtered oscillations. For each additional order the order of the iterated commutator (cf. (20)) will thereby increase in the local approximation error.

\section{FIRST-ORDER SCHEME}

We will build our numerical schemes on iterations of (5). In each iteration we embed the dominant oscillatory terms - triggered by the operator $\mathcal{L}$ - into our discretisation.

In this Section we give the main idea behind the expression of the first-order Duhamel integrator (6) presented in Section 2 and estimate its local error. We start with a trivial but important lemma on the first iteration.

Lemma 6 (First-order iteration). We have

$$
u(t)=u_{1}(t)+\mathcal{R}_{1,0}(t, u)
$$

with the first-order iteration of Duhamel's formula $u_{1}(t)$ given by

$$
u_{1}(t)=e^{t \mathcal{L}} u_{0}+\int_{0}^{t} e^{(t-\xi) \mathcal{L}} f\left(e^{\xi \mathcal{L}} u_{0}, e^{\xi \overline{\mathcal{L}}} \bar{u}_{0}\right) d \xi
$$


and the remainder

$$
\mathcal{R}_{1,0}(t, u)=\int_{0}^{t} e^{(t-\xi) \mathcal{L}}\left(f(u(\xi), \bar{u}(\xi))-f\left(e^{\xi \mathcal{L}} u_{0}, e^{\xi \overline{\mathcal{L}}} \bar{u}_{0}\right)\right) d \xi
$$

Proof. The assertion directly follows from (5).

The expansion (32) motivates the following definition,

$$
\operatorname{Osc}(t, \mathcal{L}, v, \bar{v})=\int_{0}^{t} e^{(t-\xi) \mathcal{L}} f\left(e^{\xi \mathcal{L}} v, e^{\xi \overline{\mathcal{L}}} \bar{v}\right) d \xi
$$

so that $u_{1}(t)$ defined in (32) can be expressed as

$$
u_{1}(t)=e^{t \mathcal{L}} u_{0}+\operatorname{Osc}\left(t, \mathcal{L}, u_{0}, \bar{u}_{0}\right) .
$$

In order to allow for a low regularity approximation to $u_{1}(t)$ it is thus central to find a suitable discretisation of the integral (34). For this purpose we set

$$
\mathcal{F}(t, \xi, v, \bar{v})=e^{(t-\xi) \mathcal{L}} f\left(e^{\xi \mathcal{L}} v, e^{\xi \overline{\mathcal{L}}} \bar{v}\right)
$$

such that by the fundamental theorem of calculus we have

$$
\begin{aligned}
\operatorname{Osc}(t, \mathcal{L}, v, \bar{v}) & =\int_{0}^{t} \mathcal{F}(t, \xi, v, \bar{v}) d \xi=t \mathcal{F}(t, 0, v, \bar{v})+\int_{0}^{t} \int_{0}^{\xi} \partial_{s} \mathcal{F}(t, s, v, \bar{v}) d s d \xi \\
& =t e^{t \mathcal{L}} f(v, \bar{v})+\int_{0}^{t} \int_{0}^{\xi} \partial_{s} \mathcal{F}(t, s, v, \bar{v}) d s d \xi
\end{aligned}
$$

Next we calculate that

$$
\begin{aligned}
& \partial_{\xi} \mathcal{F}(t, \xi, v, \bar{v}) \\
& \quad=e^{(t-\xi) \mathcal{L}}\left[-\mathcal{L}\left(f\left(e^{\xi \mathcal{L}} v, e^{\xi \overline{\mathcal{L}}} \bar{v}\right)\right)+D_{1} f\left(e^{\xi \mathcal{L}} v, e^{\xi \overline{\mathcal{L}}} \bar{v}\right) \cdot \mathcal{L} e^{\xi \mathcal{L}} v+D_{2} f\left(e^{\xi \mathcal{L}} v, e^{\xi \overline{\mathcal{L}}} \bar{v}\right) \cdot \overline{\mathcal{L}} e^{\xi \overline{\mathcal{L}}} \bar{v}\right]
\end{aligned}
$$

which yields thanks to our definition (8) that

$$
\partial_{\xi} \mathcal{F}(t, \xi, v, \bar{v})=e^{(t-\xi) \mathcal{L}}\left(D_{2} f\left(e^{\xi \mathcal{L}} v, e^{\xi \overline{\mathcal{L}}_{\bar{v}}}\right) \cdot \mathcal{A} e^{\xi \overline{\mathcal{L}}} \bar{v}+\mathcal{C}[f, \mathcal{L}]\left(e^{\xi \mathcal{L}} v, e^{\xi \overline{\mathcal{L}}} \bar{v}\right)\right)
$$

Remark 7. Let us observe that

- When $\mathcal{A}=0$ (which is the case in the real parabolic setting) or when $f(v, \bar{v})=f(v)$, we obtain by plugging (37) into (36) that

$$
\begin{aligned}
\left\|\operatorname{Osc}(t, \mathcal{L}, v, \bar{v})-t e^{t \mathcal{L}} f(v, \bar{v})\right\| & \leq C t^{2} \sup _{s \in[0, t]}\left\|\partial_{s} \mathcal{F}(t, s, v, \bar{v})\right\| \\
& \leq C t^{2} \sup _{\xi \in[0, t]}\left\|\mathcal{C}[f, \mathcal{L}]\left(e^{\xi \mathcal{L}} v, e^{\xi \overline{\mathcal{L}}} \bar{v}\right)\right\| .
\end{aligned}
$$

Therefore, by using Assumption 2.1, we can control the remainder if $v$ is only in $X^{\alpha_{1}}$.

- In the dispersive setting, on the other hand, where $\mathcal{L}=i \mathcal{M}$ with $\mathcal{M}=\overline{\mathcal{M}}$ the standard Taylor series expansion of the oscillations (36) introduces a classical local error structure involving the full differential operator $\mathcal{L}$ (similarly to splitting or exponential integrator methods). Indeed, since we now have $\mathcal{A}=-2 \mathcal{L}$, we still need that $v \in X^{1}=\mathcal{D}(\mathcal{L})$ to estimate the first term in the right hand side of (37), i.e., the term $\mathcal{A} e^{\xi \overline{\mathcal{L}}} \bar{v}$.

We shall thus now develop a first-order approximation which allows low regularity approximations also in the dispersive setting $\mathcal{A}=-2 \mathcal{L}$. 
3.1. First-order approximation. To allow also in the dispersive setting for low regularity approximations we need to tackle those oscillations of (34) which produce the higher order term in (37), namely

$$
D_{2} f\left(e^{\xi \mathcal{L}} v, e^{\xi \overline{\mathcal{L}}} \bar{v}\right) \cdot \mathcal{A} e^{\xi \overline{\mathcal{L}}} \bar{v}
$$

In order to achieve this, we manipulate the principal oscillations (34) as follows. We write

$$
\operatorname{Osc}(t, \mathcal{L}, v, \bar{v})=\int_{0}^{t} e^{(t-\xi) \mathcal{L}} f\left(e^{\xi \mathcal{L}} v, e^{\xi \mathcal{L}}\left[e^{\xi \mathcal{A}} \bar{v}\right]\right) d \xi
$$

recall that $\mathcal{A}=-\mathcal{L}+\overline{\mathcal{L}}$, and define the filtered function

$$
\mathcal{N}(t, s, \xi, v, \bar{v})=e^{(t-s) \mathcal{L}} f\left(e^{s \mathcal{L}} v, e^{s \mathcal{L}} e^{\xi \mathcal{A}} \bar{v}\right) .
$$

Note that the principal oscillations (34) can be expressed with the aid of the filter function $\mathcal{N}$ as

$$
\operatorname{Osc}(t, \mathcal{L}, v, \bar{v})=\int_{0}^{t} \mathcal{N}(t, \xi, \xi, v, \bar{v}) d \xi
$$

Now the Taylor series expansion of the filtered function $\mathcal{N}(t, s, \xi, v, \bar{v})$ around $s=0$ yields that

$$
\operatorname{Osc}(t, \mathcal{L}, v, \bar{v})=\int_{0}^{t} \mathcal{N}(t, 0, \xi, v, \bar{v}) d \xi+\int_{0}^{t} \int_{0}^{\xi} \partial_{s} \mathcal{N}(t, s, \xi, v, \bar{v}) d s d \xi
$$

and we observe that thanks to the filtered structure of $\mathcal{N}$ its derivative $\partial_{s} \mathcal{N}$ introduces an improved error structure, namely

$$
\partial_{s} \mathcal{N}(t, s, \xi, v, \bar{v})=e^{(t-s) \mathcal{L}} \mathcal{C}[f, \mathcal{L}]\left(e^{s \mathcal{L}} v, e^{s \mathcal{L}} e^{\xi \mathcal{A}} \bar{v}\right)
$$

The latter can be controlled using Assumption 2.1. This motivates the following expansion of the principal oscillatory integral (36) which builds the basis of our first-order Duhamel integrator (6).

Corollary 8. It holds that

$$
O s c(t, \mathcal{L}, v, \bar{v})=e^{t \mathcal{L}} \int_{0}^{t} f\left(v, e^{\xi \mathcal{A}} \bar{v}\right) d \xi+\mathcal{R}_{1,1}(t)
$$

with the remainder

$$
\mathcal{R}_{1,1}(t)=\int_{0}^{t} \int_{0}^{\xi} e^{(t-s) \mathcal{L}} \mathcal{C}[f, \mathcal{L}]\left(e^{s \mathcal{L}} v, e^{s \mathcal{L}} e^{\xi \mathcal{A}} \bar{v}\right) d s d \xi
$$

where $\mathcal{C}[f, \mathcal{L}]$ is defined in $(8)$.

Proof. The assertion just follows from (40) together with (41) noting that

$$
\mathcal{N}(t, 0, \xi, v, \bar{v})=e^{t \mathcal{L}} f\left(v, e^{\xi \mathcal{A}} \bar{v}\right) .
$$

Thanks to Corollary 8, it remains to derive a suitable discretisation of the integral

$$
\int_{0}^{t} f\left(v, e^{\xi \mathcal{A}} \bar{v}\right) d \xi
$$

which can still have high oscillations, e.g., in the dispersive setting $\mathcal{A} \neq 0$. 
Remark 9. Again, when $f$ is independent of $\bar{v}$ or when $\mathcal{A}=0$, no additional approximation has to be carried out since

$$
\int_{0}^{t} f\left(v, e^{\xi \mathcal{A}} \bar{v}\right) d \xi=t f(v, \bar{v})
$$

On the other hand, when $\mathcal{A} \neq 0$ we need to carefully embed the remaining oscillations $e^{\xi \mathcal{A}}$ in (44) into our numerical discretisation as a simple Taylor series expansion of the latter would produce an error term again involving the full operator $\mathcal{L}$ since

$$
e^{\xi \mathcal{A}}=e^{-2 i \xi \mathcal{L}}=1+\mathcal{O}(\xi \mathcal{L})
$$

With an expansion of type (45) we would in particular come back to a classical local error structure, similar to the one of splitting or exponential integrator methods, without any improvement in regularity.

On the other hand, in the more difficult dispersive setting $\mathcal{A} \neq 0$, the advantage is that $\mathcal{A}$ generates not only a semigroup, but a group and hence we may go forward and backward in time when approximating the remaining oscillations (44). The latter is the motivation for our Assumption 1.1. We shall also begin to use here our Assumption on the structure of the nonlinearity (3).

Lemma 10. Under Assumption 1.1 it holds that

$$
\int_{0}^{t} f\left(v, e^{\xi \mathcal{A}} \bar{v}\right) d \xi=t \mathcal{B}\left(F(v) \cdot \varphi_{1}(t \mathcal{A}) G(\bar{v})\right)+\mathcal{R}_{1,2}(t)
$$

with $\varphi_{1}(z)=\frac{1}{z}\left(e^{z}-1\right)$ and the remainder

$$
\mathcal{R}_{1,2}(t)=\int_{0}^{t} \int_{0}^{\xi} \mathcal{B}\left(F(v) \cdot e^{(\xi-s) \mathcal{A}} \mathcal{C}[G, \mathcal{A}]\left(e^{s \mathcal{A}} \bar{v}\right)\right) d s d \xi .
$$

Proof. Thanks to the structure of the nonlinearity (3) (in particular using that $\mathcal{B}$ is linear) we have

$$
\int_{0}^{t} f\left(v, e^{\xi \mathcal{A}} \bar{v}\right) d \xi=\int_{0}^{t} \mathcal{B}\left(F(v) \cdot G\left(e^{\xi \mathcal{A}} \bar{v}\right)\right) d \xi
$$

Now we apply the same trick as before to filter out the dominant oscillations in $G$ (triggered by $\mathcal{A})$. For this purpose we set

$$
\mathcal{N}_{2}(\xi, \mathcal{A}, v)=e^{-\xi \mathcal{A}} G\left(e^{\xi \mathcal{A}} \bar{v}\right)
$$

(which means since $G(\cdot)$ is a vector that $e^{-\xi \mathcal{A}}$ is applied to each component) such that

$$
\mathcal{B}\left(F(v) \cdot G\left(e^{\xi \mathcal{A}} \bar{v}\right)\right)=\mathcal{B}\left(F(v) \cdot e^{\xi \mathcal{A}} \mathcal{N}_{2}(\xi, \mathcal{A}, v)\right)
$$

In this notation we obtain (using again that $\mathcal{B}$ is linear) that

$$
\begin{aligned}
\int_{0}^{t} f\left(v, e^{\xi \mathcal{A}} \bar{v}\right) d \xi & =\int_{0}^{t} \mathcal{B}\left(F(v) \cdot e^{\xi \mathcal{A}} \mathcal{N}_{2}(\xi, \mathcal{A}, v)\right) d \xi \\
& =\int_{0}^{t} \mathcal{B}\left(F(v) \cdot e^{\xi \mathcal{A}}\left[\mathcal{N}_{2}(0, \mathcal{A}, v)+\int_{0}^{\xi} \partial_{s} \mathcal{N}_{2}(s, \mathcal{A}, v) d s\right]\right) d \xi \\
& =t \mathcal{B}\left(F(v) \cdot \varphi_{1}(t \mathcal{A}) G(\bar{v})\right)+\int_{0}^{t} \int_{0}^{\xi} \mathcal{B}\left(F(v) \cdot e^{\xi \mathcal{A}} \partial_{s} \mathcal{N}_{2}(s, \mathcal{A}, v) d s\right) d \xi
\end{aligned}
$$

Using that $\partial_{s} \mathcal{N}_{2}(s, \mathcal{A}, v)=e^{-s \mathcal{A}} \mathcal{C}[G, \mathcal{A}]\left(e^{s \mathcal{A}} \bar{v}\right)$ yields the remainder $(47)$.

Collecting the results in Lemma 6, Corollary 8 and Lemma 10 we thus obtain the following first-order low regularity approximation to the exact solution $u$ of (1). 
Corollary 11. The exact solution $u$ of (1) can be expanded as

$$
u\left(t_{n}+\tau\right)=e^{\tau \mathcal{L}}\left(u\left(t_{n}\right)+\tau \mathcal{B}\left(F\left(u\left(t_{n}\right)\right) \cdot \varphi_{1}(\tau \mathcal{A}) G\left(\overline{u\left(t_{n}\right)}\right)\right)\right)+\mathcal{R}_{1}\left(\tau, t_{n}\right)
$$

where the remainder $\mathcal{R}_{1}\left(\tau, t_{n}\right)$ is given by

$$
\begin{aligned}
\mathcal{R}_{1}\left(\tau, t_{n}\right) & =\int_{0}^{\tau} e^{(\tau-\xi) \mathcal{L}}\left(f\left(u\left(t_{n}+\xi\right), \bar{u}\left(t_{n}+\xi\right)\right)-f\left(e^{\xi \mathcal{L}} u\left(t_{n}\right), e^{\xi \overline{\mathcal{L}}} \bar{u}\left(t_{n}\right)\right)\right) d \xi \\
& +\int_{0}^{t} \int_{0}^{\xi} e^{(t-s) \mathcal{L}} \mathcal{C}[f, \mathcal{L}]\left(e^{s \mathcal{L}} v, e^{s \mathcal{L}} e^{\xi \mathcal{A}} \bar{v}\right) d s d \xi \\
& +e^{\tau \mathcal{L}} \int_{0}^{\tau} \int_{0}^{\xi} \mathcal{B}\left(F\left(u\left(t_{n}\right)\right) \cdot e^{(\xi-s) \mathcal{A}} \mathcal{C}[G, \mathcal{A}]\left(e^{s \mathcal{A}} \bar{u}\left(t_{n}\right)\right)\right) d s d \xi .
\end{aligned}
$$

Corollary 11 motivates the first-order scheme defined in (6) which locally yields a low-regularity second-order approximation to the exact solution $u(t)$ of $(1)$ at time $t=t_{\ell+1}=\tau \cdot(\ell+1)$. The precise error estimates will be given in Section 3.2 below.

Remark 12. Note that in some cases the approximation of the underlying oscillatory structure of (1) can be even improved. We illustrate this on the example of the KdV equation. For the KdV equation the principal oscillatory integral (34) takes thanks to the expansion (36) the form

$$
\begin{aligned}
\operatorname{Osc}(t, \mathcal{L}, v) & =t \partial_{x} v^{2}+3 \int_{0}^{t} \int_{0}^{\xi} e^{s \partial_{x}^{3}} \partial_{x}^{2}\left(e^{-s \partial_{x}^{3}} \partial_{x} v\right)^{2} \\
& =t \partial_{x} v^{2}+3 \partial_{x} \int_{0}^{t} \operatorname{Osc}\left(\xi, \mathcal{L}, \partial_{x} v\right) d \xi
\end{aligned}
$$

Taking the derivative with respect to time $t$ of the above relation we observe that

$$
\operatorname{Osc}\left(t, \mathcal{L}, \partial_{x} v\right)=\partial_{x}^{-1} \partial_{t} \operatorname{Osc}(t, \mathcal{L}, v)-v^{2} .
$$

This implies

$$
\begin{aligned}
\operatorname{Osc}(t, \mathcal{L}, v) & =\frac{1}{3} \partial_{x}^{-1} \partial_{t} \operatorname{Osc}\left(t, \mathcal{L}, \partial_{x}^{-1} v\right)-\frac{1}{3}\left(\partial_{x}^{-1} v\right)^{2} \\
& =\frac{1}{3} e^{-t \partial_{x}^{2}}\left(e^{t \partial_{x}^{2}} \partial_{x}^{-1} v\right)^{2}-\frac{1}{3}\left(\partial_{x}^{-1} v\right)^{2}
\end{aligned}
$$

Together with Lemma 6, we hence recover a low regularity approximation to the KdV equation which in the periodic setting collapses exactly to the resonance based scheme proposed in [14]. For optimal second order schemes for periodic $\mathrm{KdV}$ we refer to [28, 5].

\subsection{Local error estimates.}

Proposition 13 (Local error estimates). Let us define

$$
\mathcal{E}\left(\tau, t_{n}\right)=u\left(t_{n+1}\right)-\Phi_{\text {num }, 1}^{\tau}\left(u\left(t_{n}\right)\right) .
$$

Then we have

$$
\mathcal{E}\left(\tau, t_{n}\right)=\mathcal{E}_{1}\left(\tau, t_{n}\right)+\mathcal{E}_{2}\left(\tau, t_{n}\right)+\mathcal{E}_{3}\left(\tau, t_{n}\right)
$$

with

$$
\begin{aligned}
& \mathcal{E}_{1}\left(\tau, t_{n}\right)=\int_{0}^{\tau} e^{(\tau-\xi) \mathcal{L}}\left(f\left(u\left(t_{n}+\xi\right), \bar{u}\left(t_{n}+\xi\right)\right)-f\left(e^{\xi \mathcal{L}} u\left(t_{n}\right), e^{\xi \overline{\mathcal{L}}} \bar{u}\left(t_{n}\right)\right)\right) d \xi \\
& \mathcal{E}_{2}\left(\tau, t_{n}\right)=\int_{0}^{t} \int_{0}^{\xi} e^{(t-s) \mathcal{L}} \mathcal{C}[f, \mathcal{L}]\left(e^{s \mathcal{L}} v, e^{s \mathcal{L}} e^{\xi \mathcal{A}} \bar{v}\right) d s d \xi \\
& \mathcal{E}_{3}\left(\tau, t_{n}\right)=e^{\tau \mathcal{L}} \int_{0}^{\tau} \int_{0}^{\xi} \mathcal{B}\left(F\left(u\left(t_{n}\right)\right) \cdot e^{(\xi-s) \mathcal{A}} \mathcal{C}[G, \mathcal{A}]\left(e^{s \mathcal{A}} \bar{u}\left(t_{n}\right)\right)\right) d s d \xi
\end{aligned}
$$


If Assumption 1.1, 1.2, 2.1, 2.2 hold and assuming that

$$
\sup _{[0, T]}\|u(t)\|_{\alpha_{1}} \leq C_{T}
$$

(where $\alpha_{1}$ given by Assumption 2.1) for some $C_{T}>0$, then there exists $M_{T}>0$ such that for every $\tau \in(0,1]$

for some $\epsilon>0$.

$$
\left\|\mathcal{E}\left(\tau, t_{n}\right)\right\|_{\alpha_{0}} \leq M_{T} \tau^{2}, \quad\left\|\mathcal{E}\left(\tau, t_{n}\right)\right\|_{a_{0}} \leq M_{T} \tau^{1+\epsilon}, \quad 0 \leq n \tau \leq T
$$

Proof. Within the proof $M_{T}$ will stand for a harmless number that depends only on $C_{T}$ which changes from line to line. We first estimate $\mathcal{E}_{1}\left(\tau, t_{n}\right)$. By using Assumption 1.1 and (12), we get that

$$
\left\|\mathcal{E}_{1}\left(\tau, t_{n}\right)\right\|_{\alpha_{0}} \leq M_{T} \tau \sup _{\left[t_{n}, t_{n}+\tau\right]}\left\|u\left(t_{n}+\xi\right)-e^{\xi \mathcal{L}} u\left(t_{n}\right)\right\|_{\alpha_{0}}
$$

Next by using again the Duhamel formula (5) and Assumption with the second part of (12), we get that

thanks to (54). This yields

$$
\left\|u\left(t_{n}+\xi\right)-e^{\xi \mathcal{L}} u\left(t_{n}\right)\right\|_{\alpha_{0}} \leq M_{T} \tau
$$

$$
\left\|\mathcal{E}_{1}\left(\tau, t_{n}\right)\right\|_{\alpha_{0}} \leq M_{T} \tau^{2}
$$

The estimate of $\mathcal{E}_{2}\left(\tau, t_{n}\right)$ directly follows from Assumption 1.1 and Assumption 2.1 (cf. (10)). We get thanks to (54) that

$$
\left\|\mathcal{E}_{2}\left(\tau, t_{n}\right)\right\|_{\alpha_{0}} \leq \tau^{2} M_{T}
$$

In a similar way, by using Assumption 2.1, (11) we get that

$$
\left\|\mathcal{E}_{3}\left(\tau, t_{n}\right)\right\|_{\alpha_{0}} \leq \tau^{2} M_{T}
$$

Consequently, by combining the last estimate and (55), (56), we get that

$$
\left\|\mathcal{E}\left(\tau, t_{n}\right)\right\|_{\alpha_{0}} \leq M_{T} \tau^{2} .
$$

It remains to estimate $\left\|\mathcal{E}\left(\tau, t_{n}\right)\right\|_{a_{0}}$. We observe that thanks to (50), the Duhamel formula (5) and (6) we can write

$$
\begin{aligned}
\mathcal{E}\left(\tau, t_{n}\right) & =\int_{0}^{\tau} e^{(\tau-s) \mathcal{L}} f\left(u\left(t_{n}+s\right), \bar{u}\left(t_{n}+s\right)\right) d s-\tau e^{\tau \mathcal{L}} \mathcal{B}\left(F\left(u\left(t_{n}\right)\right) \varphi_{1}(\tau \mathcal{A}) G\left(\bar{u}\left(t_{n}\right)\right)\right) \\
& =\int_{t_{n}}^{t_{n+1}} e^{\left(t_{n+1}-s\right) \mathcal{L}} f(u(s), \bar{u}(s)) d s-\tau e^{\tau \mathcal{L}} \mathcal{B}\left(F\left(u\left(t_{n}\right)\right) \varphi_{1}(\tau \mathcal{A}) G\left(\bar{u}\left(t_{n}\right)\right)\right) .
\end{aligned}
$$

We then estimate the two terms in the above right hand side separately in $X^{\alpha_{1}}$ by using (54) and Assumption 1.2 for the first one (recall that $\alpha_{1} \in\left(a_{0}, a_{1}\right]$ ) and (13) for the second one. This yields

$$
\left\|\mathcal{E}\left(\tau, t_{n}\right)\right\|_{\alpha_{1}} \leq \tau M_{T}
$$

To conclude for the estimate of $\left\|\mathcal{E}\left(\tau, t_{n}\right)\right\|_{a_{0}}$, we observe that if $\alpha_{0} \geq a_{0}$, we can just use the continuous embedding $X^{\alpha_{0}} \subset X^{a_{0}}$ and hence take $\epsilon=1$. If $\alpha_{0}<a_{0}$, since we always assume that $\alpha_{1}>a_{0}$, we can use the interpolation properties of the $X^{\alpha}$ spaces. In particular, we obtain that

$$
\left\|\mathcal{E}\left(\tau, t_{n}\right)\right\|_{a_{0}} \leq\left\|\mathcal{E}\left(\tau, t_{n}\right)\right\|_{\alpha_{1}}^{\theta}\left\|\mathcal{E}\left(\tau, t_{n}\right)\right\|_{\alpha_{0}}^{1-\theta}
$$

for some $\theta \in(0,1)$. Therefore, thanks to (58), (57), we obtain that

$$
\left\|\mathcal{E}\left(\tau, t_{n}\right)\right\|_{a_{0}} \leq M_{T} \tau^{\theta}\left(\tau^{2}\right)^{1-\theta} \leq M_{T} \tau^{2-\theta}
$$

which is the desired estimate since $2-\theta>1$.

With the above proposition at hand we can prove our global error estimate in Theorem 2. 
3.3. Proof of Theorem 2. Let us set $e^{n}=u\left(t_{n}\right)-u^{n}, u^{n}$ being the numerical solution computed with (6). By using Proposition 13, we get that

$$
e^{n+1}=\Phi_{n u m, 1}^{\tau}\left(u\left(t_{n}\right)\right)-\Phi_{n u m, 1}^{\tau}\left(u^{n}\right)+\mathcal{E}\left(\tau, t_{n}\right), \quad e^{0}=0 .
$$

By using the expression of the scheme, we get that

$$
e^{n+1}=e^{\tau \mathcal{L}}\left[e^{n}+\tau\left(\Psi_{\text {num }, 1}^{\tau}\left(u\left(t_{n}\right)\right)-\Psi_{\text {num }, 1}^{\tau}\left(u^{n}\right)\right)\right]+\mathcal{E}\left(\tau, t_{n}\right) .
$$

By using (14) and Proposition 13, we get that for $0 \leq n \tau \leq T$,

$$
\left\|e^{n+1}\right\|_{\alpha_{0}} \leq\left\|e^{n}\right\|_{\alpha_{0}}+\tau C_{\alpha_{0}}\left(\left\|u\left(t_{n}\right)\right\|_{a_{0}},\left\|e^{n}\right\|_{a_{0}}\right)\left\|e^{n}\right\|_{\alpha_{0}}+M_{T} \tau^{2} .
$$

In a similar way, by using (15), and Proposition 13, we obtain that

$$
\left\|e^{n+1}\right\|_{a_{0}} \leq\left\|e^{n}\right\|_{a_{0}}+\tau C_{a_{0}}\left(\left\|u\left(t_{n}\right)\right\|_{a_{0}},\left\|e^{n}\right\|_{a_{0}}\right)\left\|e^{n}\right\|_{a_{0}}+M_{T} \tau^{1+\epsilon} .
$$

We then easily get by induction that for $\tau$ sufficiently small, we have that

$$
\sup _{0 \leq n \leq N}\left\|e^{n}\right\|_{a_{0}}<+\infty, \quad \sup _{0 \leq n \leq N}\left\|e^{n}\right\|_{\alpha_{0}} \leq M_{T} \tau .
$$

This ends the proof.

\section{SECOND-ORDER APPROXIMATION}

To establish a second-order approximation at low regularity we need to iterate Duhamel's formula (5) up to higher order and subsequently embed the underlying (iterated) dominant oscillations into our discretisation. Surprisingly, it turns out that the main difficulty thereby lies in providing a higher order discretisation of the principal oscillations (34).

We commence with a Lemma on the second-order iteration of Duhamel's formula.

Lemma 14. At second-order we have

$$
u(t)=u_{2}(t)+\mathcal{R}_{2,0}(t)
$$

with the second-oder iteration of Duhamel's formula given by

$$
u_{2}(t)=e^{t \mathcal{L}} u_{0}+\operatorname{Osc}\left(t, \mathcal{L}, u_{0}, \bar{u}_{0}\right)+\frac{t^{2}}{2} e^{t \mathcal{L}}\left(D_{1} f\left(u_{0}, \bar{u}_{0}\right) \cdot f\left(u_{0}, \bar{u}_{0}\right)+D_{2} f\left(u_{0}, \bar{u}_{0}\right) \cdot \overline{f\left(u_{0}, \bar{u}_{0}\right)}\right),
$$

where the principal oscillations $\operatorname{Osc}\left(t, \mathcal{L}, u_{0}, \bar{u}_{0}\right)$ are defined in $(34)$ and the remainder $\mathcal{R}_{2,0}(t)$ takes the form

$$
\begin{gathered}
\mathcal{R}_{2,0}(t)=\int_{0}^{t} e^{(t-\xi) \mathcal{L}}\left[f\left(e^{\xi \mathcal{L}} u_{0}+\xi f\left(u_{0}, \bar{u}_{0}\right)+\mathcal{R}_{2, a}(0, \xi), e^{\xi \overline{\mathcal{L}}} \bar{u}_{0}+\xi \overline{f\left(u_{0}, \bar{u}_{0}\right)}+\overline{\mathcal{R}_{2, a}}(0, \xi)\right)\right. \\
\left.-f\left(e^{\xi \mathcal{L}} u_{0}, e^{\xi \overline{\mathcal{L}}} \bar{u}_{0}\right)\right] d \xi \\
-e^{t \mathcal{L}} \int_{0}^{t} \xi\left(D_{1} f\left(u_{0}, \bar{u}_{0}\right) \cdot f\left(u_{0}, \bar{u}_{0}\right)+D_{2} f\left(u_{0}, \bar{u}_{0}\right) \cdot \overline{f\left(u_{0}, \bar{u}_{0}\right)}\right) d \xi
\end{gathered}
$$

with

$$
\mathcal{R}_{2, a}\left(t_{n}, \xi\right)=\int_{0}^{\xi} e^{(\xi-s) \mathcal{L}} f\left(u\left(t_{n}+s\right), \bar{u}\left(t_{n}+s\right)\right) d s-\xi f\left(u\left(t_{n}\right), \bar{u}\left(t_{n}\right)\right) .
$$

Proof. First note that by replacing $t$ with $\xi$ in (5) we have that

$$
u(\xi)=e^{\xi \mathcal{L}} u_{0}+\int_{0}^{\xi} e^{(\xi-s) \mathcal{L}} f(u(s), \bar{u}(s)) d s=e^{\xi \mathcal{L}} u_{0}+\xi f\left(u_{0}, \bar{u}_{0}\right)+\mathcal{R}_{2, a}(0, \xi)
$$


with the remainder $\mathcal{R}_{2, a}(0, \xi)$ given in (63). The latter is thereby formally of order $\mathcal{O}\left(\xi^{2} \mathcal{L} f(u, \bar{u})\right)$. The proof then follows by iterating Duhamel's formula, i.e., plugging the expansion (64) into (5), which yields that

$$
u(t)=e^{t \mathcal{L}} u_{0}+\int_{0}^{t} e^{(t-\xi) \mathcal{L}} f\left(e^{\xi \mathcal{L}} u_{0}+\xi f\left(u_{0}, \bar{u}_{0}\right)+\mathcal{R}_{2, a}(0, \xi), e^{\xi \overline{\mathcal{L}}_{u_{0}}+\xi \overline{f\left(u_{0}, \bar{u}_{0}\right)}}+\overline{\mathcal{R}_{2, a}}(0, \xi)\right) d \xi .
$$

Thanks to Lemma 14 it thus remains to derive a suitable second-order approximation to the principal oscillations (cf. (34))

$$
\operatorname{Osc}\left(t, \mathcal{L}, u_{0}, \bar{u}_{0}\right)=\int_{0}^{t} e^{(t-\xi) \mathcal{L}} f\left(e^{\xi \mathcal{L}} u_{0}, e^{\xi \overline{\mathcal{L}}_{\bar{u}}}\right) d \xi
$$

In contrast to the first-order approximation discussed in Section 3 we now have to embed iterations of these oscillations into our discretisation. Following (39) we rewrite the principal oscillations with the aid of of the filtered function

$$
\mathcal{N}(t, s, \xi, v, \bar{v})=e^{(t-s) \mathcal{L}} f\left(e^{s \mathcal{L}} v, e^{s \mathcal{L}} e^{\xi \mathcal{A}} \bar{v}\right)=e^{(t-s) \mathcal{L}} \mathcal{B}\left(F\left(e^{s \mathcal{L}} v\right) \cdot G\left(e^{s \mathcal{L}} e^{\xi \mathcal{A}} \bar{v}\right)\right) .
$$

With this notation at hand we have that

$$
\operatorname{Osc}(t, \mathcal{L}, v, \bar{v})=\int_{0}^{t} \mathcal{N}(t, \xi, \xi, v, \bar{v}) d \xi .
$$

Next we carry out a Taylor series expansion up to second-order of the filtered function $\mathcal{N}(t, s, \xi, v, \bar{v})$ defined in (65) around $s=0$. With the the notation $\partial_{s} \mathcal{N}(t, 0, \xi, v, \bar{v})=\left.\partial_{s} \mathcal{N}(t, s, \xi, v, \bar{v})\right|_{s=0}$ this yields that

$$
\mathcal{N}(t, s, \xi, v, \bar{v})=\mathcal{N}(t, 0, \xi, v, \bar{v})+\xi \partial_{s} \mathcal{N}(t, 0, \xi, v, \bar{v})+\int_{0}^{\xi} \int_{0}^{s} \partial_{s_{1}}^{2} \mathcal{N}\left(t, s_{1}, \xi, v, \bar{v}\right) d s_{1} d s
$$

where $\partial_{s_{1}}^{2} \mathcal{N}\left(t, s_{1}, \xi, v, \bar{v}\right)$ obeys the improved error structure

$$
\partial_{s}^{2} \mathcal{N}(t, s, \xi, v, \bar{v})=e^{(t-s) \mathcal{L}} \mathcal{C}^{2}[f, \mathcal{L}]\left(e^{s \mathcal{L}} v, e^{s \mathcal{L}} e^{\xi \mathcal{A}} \bar{v}\right)
$$

where we recall that

$$
\mathcal{C}^{2}[f, \mathcal{L}]=\mathcal{C}[\mathcal{C}[f, \mathcal{L}], \mathcal{L}]
$$

Together with (66) we thus obtain

$$
\begin{aligned}
\operatorname{Osc}(t, \mathcal{L}, v, \bar{v}) & =\int_{0}^{t} \mathcal{N}(t, 0, \xi, v, \bar{v}) d \xi+\int_{0}^{t} \xi \partial_{s} \mathcal{N}(t, 0, \xi, v, \bar{v}) d \xi \\
& +\int_{0}^{t} \int_{0}^{\xi} \int_{0}^{s} e^{\left(t-s_{1}\right) \mathcal{L}} \mathcal{C}^{2}[f, \mathcal{L}]\left(e^{s_{1} \mathcal{L}} v, e^{s_{1} \mathcal{L}} e^{\xi \mathcal{A}} \bar{v}\right) d s_{1} d s d \xi .
\end{aligned}
$$

Note that in the expansion (68) we will have to include the term $\partial_{s} \mathcal{N}(t, 0, \xi, v, \bar{v})$ explicitly in our scheme. Thus, in order to guarante stability of the scheme we next exploit that formally we have for some $0 \leq \eta \leq t$ that

$$
\partial_{s} \mathcal{N}(t, 0, \xi, v, \bar{v})=\left.\frac{\delta_{\zeta} \mathcal{N}(t, \zeta, \xi, v, \bar{v})}{t}\right|_{\zeta=t}+\mathcal{O}\left(t \partial_{\zeta}^{2} \mathcal{N}(t, \eta, \xi, v, \bar{v})\right)
$$

with the standard shift operator

$$
\delta_{\zeta} g(\zeta):=g(\zeta)-g(0)
$$

The latter motivates us to further express the expansion of the oscillations (68) as follows

$$
\operatorname{Osc}(t, \mathcal{L}, v, \bar{v})=\int_{0}^{t} \mathcal{N}(t, 0, \xi, v, \bar{v}) d \xi+\left.\int_{15}^{t} \xi \frac{\delta_{\zeta} \mathcal{N}(t, \zeta, \xi, v, \bar{v})}{t}\right|_{\zeta=t} d \xi+\mathcal{R}_{\mathcal{N}}(t)
$$


(see also [5]) where the remainder takes the form

$$
\begin{aligned}
\mathcal{R}_{\mathcal{N}}(t)= & \int_{0}^{t} \xi\left(\partial_{s} \mathcal{N}(t, 0, \xi, v, \bar{v})-\left.\frac{\delta_{\zeta} \mathcal{N}(t, \zeta, \xi, v, \bar{v})}{t}\right|_{\zeta=t}\right) d \xi+\int_{0}^{t} \int_{0}^{\xi} \int_{0}^{s} \partial_{s_{1}}^{2} \mathcal{N}\left(t, s_{1}, \xi, v, \bar{v}\right) d s_{1} d s d \xi \\
= & -\int_{0}^{t} \xi \int_{0}^{1} \int_{0}^{t s} \partial_{s_{1}}^{2} \mathcal{N}\left(t, s_{1}, \xi, v, \bar{v}\right) d s_{1} d s d \xi+\int_{0}^{t} \int_{0}^{\xi} \int_{0}^{s} \partial_{s_{1}}^{2} \mathcal{N}\left(t, s_{1}, \xi, v, \bar{v}\right) d s_{1} d s d \xi \\
= & -\int_{0}^{t} \xi \int_{0}^{1} \int_{0}^{t s} e^{\left(t-s_{1}\right) \mathcal{L}} \mathcal{C}^{2}[f, \mathcal{L}]\left(e^{s_{1} \mathcal{L}} v, e^{s_{1} \mathcal{L}} e^{\xi \mathcal{A}} \bar{v}\right) d s_{1} d s d \xi \\
& \quad+\int_{0}^{t} \int_{0}^{\xi} \int_{0}^{s} e^{\left(t-s_{1}\right) \mathcal{L}} \mathcal{C}^{2}[f, \mathcal{L}]\left(e^{s_{1} \mathcal{L}} v, e^{s_{1} \mathcal{L}} e^{\xi \mathcal{A}} \bar{v}\right) d s_{1} d s d \xi .
\end{aligned}
$$

Remark 15. Again, by the definition of $\mathcal{N}$ in (65) we obtain for nonlinearities such that $f(v, \bar{v})=$ $f(v)$ that

$$
\mathcal{N}(t, 0, \xi, v, \bar{v})=e^{t \mathcal{L}} f(v, \bar{v}) \quad \text { and } \quad \mathcal{N}(t, t, \xi, v, \bar{v})=f\left(e^{t \mathcal{L}} v, e^{t \mathcal{L}} \bar{v}\right) \quad \text { for all } \quad \xi
$$

This also holds true when $\mathcal{A}=-\mathcal{L}+\overline{\mathcal{L}}=0$. Thus in these cases $(\mathcal{A}=0$ or $f$ independent of $\bar{v})$ we do not need to carry out any additional approximation as we simply obtain by (69) that

$$
\operatorname{Osc}(t, \mathcal{L}, v, \bar{v})=t e^{t \mathcal{L}} f(v, \bar{v})+\frac{t}{2} \delta_{t} f\left(e^{t \mathcal{L}} v, e^{t \mathcal{L}} \bar{v}\right)+\mathcal{R}_{\mathcal{N}}(t)
$$

When $\mathcal{A} \neq 0$, we still need to tackle the remaining oscillations in the integral terms of (69).

We state a lemma on the integration of the oscillations in $\xi \mathcal{N}(t, s, \xi, v, \bar{v})$ which will allow us to handle the second term in (69) also in the general setting where $\mathcal{A} \neq 0$ and $f$ depends on $\bar{v}$.

Lemma 16. Under Assumption 1.2 we have that

$$
\left.\int_{0}^{t} \xi \frac{\delta_{\zeta} \mathcal{N}(t, \zeta, \xi, v, \bar{v})}{t}\right|_{\zeta=t} d \xi=t \delta_{\xi=t} e^{(t-\xi) \mathcal{L}} \mathcal{B}\left(F\left(e^{\xi \mathcal{L}} v\right) \cdot \varphi_{2}(t \mathcal{A}) G\left(e^{\xi \mathcal{L}} \bar{v}\right)\right)+\mathcal{R}_{d \mathcal{N}_{1}}(t)
$$

where $\varphi_{2}(z)=\frac{1}{z}\left(e^{z}-\varphi_{1}(z)\right)$ and the remainder takes the form

$$
\mathcal{R}_{d \mathcal{N}_{1}}(t)=\int_{0}^{t} \int_{0}^{\xi} \frac{\xi}{t} \int_{0}^{t} e^{\left(t-s_{1}\right) \mathcal{L}} \mathcal{C}\left[\Psi_{\xi, s}, \mathcal{L}\right]\left(e^{s_{1} \mathcal{L}} v, e^{s \mathcal{A}} e^{s_{1} \mathcal{L}} \bar{v}\right) d s_{1} d s d \xi
$$

with $\Psi_{\xi, s}(v, w)=\mathcal{B}\left(F(v) \cdot e^{(\xi-s) \mathcal{A}} \mathcal{C}[G, \mathcal{A}](w)\right)$.

Proof. Note that

$$
\left.\int_{0}^{t} \xi \frac{\delta_{\zeta} \mathcal{N}(t, \zeta, \xi, v, \bar{v})}{t}\right|_{\zeta=t} d \xi=\frac{1}{t} \int_{0}^{t} \xi(\mathcal{N}(t, t, \xi, v, \bar{v})-\mathcal{N}(t, 0, \xi, v, \bar{v})) d \xi
$$


With Assumption 1.2 at hand we obtain similarly to (46) that

$$
\begin{aligned}
\int_{0}^{t} \xi \mathcal{N} & (t, t, \xi, v, \bar{v}) d \xi=\int_{0}^{t} \xi \mathcal{B}\left(F\left(e^{t \mathcal{L}} v\right) \cdot G\left(e^{t \mathcal{L}} e^{\xi \mathcal{A}} \bar{v}\right)\right) d \xi \\
= & \int_{0}^{t} \xi \mathcal{B}\left(F\left(e^{t \mathcal{L}} v\right) \cdot e^{\xi \mathcal{A}}\left[e^{-\xi \mathcal{A}} G\left(e^{t \mathcal{L}} e^{\xi \mathcal{A}} \bar{v}\right)\right]\right) d \xi \\
= & \int_{0}^{t} \xi \mathcal{B}\left(F\left(e^{t \mathcal{L}} v\right) \cdot e^{\xi \mathcal{A}}\left[G\left(e^{t \mathcal{L}} \bar{v}\right)+\int_{0}^{\xi} \partial_{s}\left(e^{-s \mathcal{A}} G\left(e^{t \mathcal{L}} e^{s \mathcal{A}} \bar{v}\right)\right) d s\right]\right) d \xi \\
= & \int_{0}^{t} \xi \mathcal{B}\left(F\left(e^{t \mathcal{L}} v\right) \cdot e^{\xi \mathcal{A}} G\left(e^{t \mathcal{L}} \bar{v}\right)\right) d \xi+\int_{0}^{t} \int_{0}^{\xi} \xi \mathcal{B}\left(F\left(e^{t \mathcal{L}} v\right) \cdot e^{(\xi-s) \mathcal{A}} \mathcal{C}[G, \mathcal{A}]\left(e^{t \mathcal{L}} e^{s \mathcal{A}} \bar{v}\right)\right) d s d \xi \\
= & t^{2} \mathcal{B}\left(F\left(e^{t \mathcal{L}} v\right) \cdot \varphi_{2}(t \mathcal{A}) G\left(e^{t \mathcal{L}} \bar{v}\right)\right) d \xi+\int_{0}^{t} \int_{0}^{\xi} \xi \mathcal{B}\left(F\left(e^{t \mathcal{L}} v\right) \cdot e^{(\xi-s) \mathcal{A}} \mathcal{C}[G, \mathcal{A}]\left(e^{t \mathcal{L}} e^{s \mathcal{A}} \bar{v}\right)\right) d s d \xi .
\end{aligned}
$$

In the same manner we obtain

$$
\begin{aligned}
\int_{0}^{t} \xi \mathcal{N}(t, 0, \xi, v, \bar{v}) d \xi=e^{t \mathcal{L}} \int_{0}^{t} \xi \mathcal{B}\left(F(v) \cdot G\left(e^{\xi \mathcal{A}} \bar{v}\right)\right) d \xi \\
\quad=t^{2} e^{t \mathcal{L}} \mathcal{B}\left(F(v) \cdot \varphi_{2}(t \mathcal{A}) G(\bar{v})\right) d \xi+e^{t \mathcal{L}} \int_{0}^{t} \int_{0}^{\xi} \xi \mathcal{B}\left(F(v) \cdot e^{(\xi-s) \mathcal{A}} \mathcal{C}[G, \mathcal{A}]\left(e^{s \mathcal{A}} \bar{v}\right)\right) d s d \xi
\end{aligned}
$$

Using that

$$
\begin{aligned}
& \int_{0}^{t} \int_{0}^{\xi} \frac{\xi}{t}\left[\mathcal{B}\left(F\left(e^{t \mathcal{L}} v\right) \cdot e^{(\xi-s) \mathcal{A}} \mathcal{C}[G, \mathcal{A}]\left(e^{t \mathcal{L}} e^{s \mathcal{A}} \bar{v}\right)\right)-e^{t \mathcal{L}} \mathcal{B}\left(F(v) \cdot e^{(\xi-s) \mathcal{A}} \mathcal{C}[G, \mathcal{A}]\left(e^{s \mathcal{A}} \bar{v}\right)\right)\right] d s d \xi \\
& =\int_{0}^{t} \int_{0}^{\xi} \frac{\xi}{t} \int_{0}^{t} \partial_{s_{1}} e^{\left(t-s_{1}\right) \mathcal{L}} \mathcal{B}\left(F\left(e^{s_{1} \mathcal{L}} v\right) \cdot e^{(\xi-s) \mathcal{A}} \mathcal{C}[G, \mathcal{A}]\left(e^{s_{1} \mathcal{L}} e^{s \mathcal{A}} \bar{v}\right)\right) d_{s_{1}} d s d \xi \\
& =\int_{0}^{t} \int_{0}^{\xi} \frac{\xi}{t} \int_{0}^{t} e^{\left(t-s_{1}\right) \mathcal{L}} \mathcal{C}\left[\Psi_{\xi, s}, \mathcal{L}\right]\left(e^{s_{1} \mathcal{L}} v, e^{s \mathcal{A}} e^{s_{1} \mathcal{L}} \bar{v}\right) d s_{1} d s d \xi
\end{aligned}
$$

with $\Psi_{\xi, s}(v, w)=\mathcal{B}\left(F(v) \cdot e^{(\xi-s) \mathcal{A}} \mathcal{C}[G, \mathcal{A}](w)\right)$ concludes the proof.

Finally, to handle the first term in (69), we need to derive a suitable second-order approximation to

$$
\int_{0}^{t} \mathcal{N}(t, 0, \xi, v, \bar{v}) d \xi=e^{t \mathcal{L}} \int_{0}^{t} \mathcal{B}\left(F(v) \cdot G\left(e^{\xi \mathcal{A}} \bar{v}\right)\right) d \xi
$$

Lemma 17. Under Assumption 1.2 we have that

$$
\begin{aligned}
\int_{0}^{t} \mathcal{N}(t, 0, \xi, v, \bar{v}) d \xi & =t e^{t \mathcal{L}} \mathcal{B}\left(F(v) \cdot \varphi_{1}(t \mathcal{A}) G(\bar{v})\right) \\
& +t e^{t \mathcal{L}} \mathcal{B}\left(F(v) \cdot \varphi_{2}(t \mathcal{A}) \delta_{t}\left(e^{-t \mathcal{A}} G\left(e^{t \mathcal{A}} \bar{v}\right)\right)\right)+\mathcal{R}_{d \mathcal{N}_{2}}(t)
\end{aligned}
$$

where the remainder $\mathcal{R}_{d \mathcal{N}}(t)$ is given by

$$
\begin{aligned}
\mathcal{R}_{d \mathcal{N}_{2}}(t) & =e^{t \mathcal{L}} \int_{0}^{t} \int_{0}^{\xi} \int_{0}^{\xi_{1}} \mathcal{B}\left(F(v) \cdot e^{\left(\xi-\xi_{2}\right) \mathcal{A}} \mathcal{C}^{2}[G, \mathcal{A}]\left(e^{\xi_{2} \mathcal{A}} \bar{v}\right)\right) d \xi_{2} d \xi_{1} d \xi \\
& +e^{t \mathcal{L}} \int_{0}^{t} \xi \int_{0}^{1} \int_{0}^{t s} \mathcal{B}\left(F(v) \cdot\left[e^{\left(\xi-s_{1}\right) \mathcal{A}} \mathcal{C}^{2}[G, \mathcal{A}]\left(e^{s_{1} \mathcal{A}} \bar{v}\right) d s_{1} d s\right]\right) d \xi
\end{aligned}
$$


Proof. We carry out a Taylor series expansion in the spirit of (46). At higher-order this yields with the notation $\mathcal{N}_{2}(t, \mathcal{A}, v)=e^{-t \mathcal{A}} G\left(e^{t \mathcal{A}} \bar{v}\right)$ that

$$
\begin{aligned}
& \int_{0}^{t} \mathcal{N}(t, 0, \xi, v, \bar{v}) d \xi=e^{t \mathcal{L}} \int_{0}^{t} \mathcal{B}\left(F(v) \cdot G\left(e^{\xi \mathcal{A}} \bar{v}\right)\right) d \xi=e^{t \mathcal{L}} \int_{0}^{t} \mathcal{B}\left(F(v) \cdot e^{\xi \mathcal{A}} \mathcal{N}_{2}(\xi, \mathcal{A}, v)\right) d \xi \\
& =e^{t \mathcal{L}} \int_{0}^{t} \mathcal{B}\left(F(v) \cdot e^{\xi \mathcal{A}}\left[G(\bar{v})+\xi\left[\partial_{\xi} \mathcal{N}_{2}(\xi, \mathcal{A}, v)\right]_{\xi=0}+\int_{0}^{\xi} \int_{0}^{\xi_{1}} \partial_{\xi_{2}}^{2} \mathcal{N}_{2}\left(\xi_{2}, \mathcal{A}, v\right) d \xi_{2} d \xi_{1}\right]\right) d \xi .
\end{aligned}
$$

Next we use that

$$
\left.\partial_{\xi} \mathcal{N}_{2}(\xi, \mathcal{A}, v)\right|_{\xi=0}-\left.\frac{1}{t} \delta_{\xi} \mathcal{N}_{2}(\xi, \mathcal{A}, v)\right|_{\xi=t}=-\int_{0}^{1} \int_{0}^{t s} \partial_{s_{1}}^{2} \mathcal{N}_{2}\left(s_{1}, \mathcal{A}, v\right) d s_{1} d s
$$

as well as

$$
\partial_{t}^{2} \mathcal{N}_{2}(t, \mathcal{A}, v)=\partial_{t}^{2}\left(e^{-t \mathcal{A}} G\left(e^{t \mathcal{A}} \bar{v}\right)\right)=e^{-t \mathcal{A}} \mathcal{C}^{2}[G, \mathcal{A}]\left(e^{t \mathcal{A}} \bar{v}\right)
$$

where $\mathcal{C}^{2}[f, \mathcal{L}]=\mathcal{C}[\mathcal{C}[f, \mathcal{L}], \mathcal{L}]$. By the linearity of $\mathcal{B}$ this yields the assertion.

Note that in the above lemma we again used a finite difference approximation for $\left[\partial_{\xi} e^{-\xi \mathcal{A}} G\left(e^{\xi \mathcal{A}} \bar{v}\right)\right]_{\xi=0}$ in order to guarantee stability of the scheme.

Collecting the results in Lemma 14 (in particular (63)), Lemma 16 and 17 yields together with (69) and (70) the following expansion of the exact solution.

Corollary 18. The exact solution $u$ of (1) allows with the notation $f^{n}=f\left(u\left(t_{n}\right), \bar{u}\left(t_{n}\right)\right)$ the expansion

$$
\begin{aligned}
u\left(t_{n}+\tau\right) & =e^{\tau \mathcal{L}} u\left(t_{n}\right)+\tau e^{\tau \mathcal{L}} \mathcal{B}\left(F\left(u\left(t_{n}\right)\right) \cdot \varphi_{1}(\tau \mathcal{A}) G\left(\bar{u}\left(t_{n}\right)\right)\right) \\
& +\tau e^{\tau \mathcal{L}} \mathcal{B}\left(F\left(u\left(t_{n}\right)\right) \cdot \varphi_{2}(\tau \mathcal{A}) \delta_{\tau}\left(e^{-\tau \mathcal{A}} G\left(e^{\tau \mathcal{A}} \bar{u}\left(t_{n}\right)\right)\right)\right) \\
& +\tau \delta_{\xi=\tau} e^{(\tau-\xi) \mathcal{L}} \mathcal{B}\left(F\left(e^{\xi \mathcal{L}} u\left(t_{n}\right)\right) \cdot \varphi_{2}(\tau \mathcal{A}) G\left(e^{\xi \mathcal{L}} \bar{u}\left(t_{n}\right)\right)\right) \\
& +\frac{\tau^{2}}{2} e^{\tau \mathcal{L}}\left(D_{1} f^{n} \cdot f^{n}+D_{2} f^{n} \cdot \overline{f^{n}}\right) \\
& +\mathcal{R}_{2}\left(\tau, t_{n}\right)
\end{aligned}
$$

where the remainder $\mathcal{R}_{2}\left(\tau, t_{n}\right)$ takes the form

$$
\begin{aligned}
\mathcal{R}_{2}\left(\tau, t_{n}\right)= & \int_{0}^{\tau} e^{(\tau-\xi) \mathcal{L}}\left[f\left(e^{\xi \mathcal{L}} u\left(t_{n}\right)+\xi f^{n}+\mathcal{R}_{2, a}\left(t_{n}, \xi\right), e^{\xi \overline{\mathcal{L}}} \bar{u}\left(t_{n}\right)+\xi \overline{f^{n}}+\overline{\mathcal{R}_{2, a}}\left(t_{n}, \xi\right)\right)\right. \\
& \left.-f\left(e^{\xi \mathcal{L}} u\left(t_{n}\right), e^{\xi \overline{\mathcal{L}}} \bar{u}\left(t_{n}\right)\right)\right] d \xi \\
& -e^{\tau \mathcal{L}} \int_{0}^{\tau} \xi\left(D_{1} f^{n} \cdot f^{n}+D_{2} f^{n} \cdot \overline{f^{n}}\right) d \xi \\
& +\int_{0}^{\tau} \xi \int_{0}^{1} \int_{0}^{\tau s} e^{\left(\tau-s_{1}\right) \mathcal{L}} \mathcal{C}^{2}[f, \mathcal{L}]\left(e^{s_{1} \mathcal{L}} u\left(t_{n}\right), e^{s_{1} \mathcal{L}} e^{\xi \mathcal{A}} \bar{u}\left(t_{n}\right)\right) d s_{1} d s d \xi \\
& +\int_{0}^{\tau} \int_{0}^{\xi} \int_{0}^{s} e^{\left(\tau-s_{1}\right) \mathcal{L}} \mathcal{C}^{2}[f, \mathcal{L}]\left(e^{s_{1} \mathcal{L}} u\left(t_{n}\right), e^{s_{1} \mathcal{L}} e^{\xi \mathcal{A}} \bar{u}\left(t_{n}\right)\right) d s_{1} d s d \xi \\
& +e^{\tau \mathcal{L}} \int_{0}^{\tau} \int_{0}^{\xi} \int_{0}^{\xi} \mathcal{B}\left(F\left(u\left(t_{n}\right)\right) \cdot e^{\left(\xi-\xi_{2}\right) \mathcal{A}} \mathcal{C}^{2}[G, \mathcal{A}]\left(e^{\xi_{2} \mathcal{A}} \bar{u}\left(t_{n}\right)\right)\right) d \xi_{2} d \xi_{1} d \xi \\
& +e^{\tau \mathcal{L}} \int_{0}^{\tau} \xi \int_{0}^{1} \int_{0}^{\tau s} \mathcal{B}\left(F\left(u\left(t_{n}\right)\right) \cdot\left[e^{\left(\xi-s_{1}\right) \mathcal{A}} \mathcal{C}^{2}[G, \mathcal{A}]\left(e^{s_{1} \mathcal{A}} \bar{u}\left(t_{n}\right)\right) d s_{1} d s\right]\right) d \xi \\
& +\int_{0}^{\tau} \int_{0}^{\xi} \frac{\xi}{\tau} \int_{0}^{\tau} e^{\left(\tau-s_{1}\right) \mathcal{L}} \mathcal{C}\left[\Psi_{\xi-s}, \mathcal{L}\right]\left(e^{s_{1} \mathcal{L}} u\left(t_{n}\right), e^{s \mathcal{A}} e^{s_{1} \mathcal{L}} \bar{u}\left(t_{n}\right)\right) d s_{1} d s d \xi
\end{aligned}
$$


with

$$
\begin{aligned}
& \mathcal{R}_{2, a}\left(t_{n}, \xi\right)=\int_{0}^{\xi} e^{(\xi-s) \mathcal{L}} f\left(u\left(t_{n}+s\right), \bar{u}\left(t_{n}+s\right)\right) d s-\xi f\left(u\left(t_{n}\right), \bar{u}\left(t_{n}\right)\right) \\
& \Psi_{\xi}(v, w)=\mathcal{B}\left(F(v) \cdot e^{\xi \mathcal{A}} \mathcal{C}[G, \mathcal{A}](w)\right), \xi \in \mathbb{R} .
\end{aligned}
$$

Corollary 18 motivates the second-order scheme (19) which locally yields a low-regularity thirdorder approximation to the exact solution $u(t)$ at time $t=t_{\ell+1}=\tau \cdot(\ell+1)$.

\subsection{Local error estimates.}

Proposition 19 (Local error estimates for the second-order scheme). Let

$$
\mathcal{R}_{2}\left(\tau, t_{n}\right)=u\left(t_{n+1}\right)-\Phi_{\text {num }, 2}^{\tau}\left(u\left(t_{n}\right)\right)
$$

with $u$ defined in Corollary 18. If Assumption 1.1, 1.2, 2.3, 2.4 hold and assuming that

$$
\sup _{[0, T]}\|u(t)\|_{\alpha_{2}} \leq C_{T}
$$

(where $\alpha_{2}$ is given by Assumption 2.3) for some $C_{T}>0$, then there exists $M_{T}>0$ such that for every $\tau \in(0,1]$

$$
\left\|\mathcal{R}_{2}\left(\tau, t_{n}\right)\right\|_{\alpha_{0}} \leq M_{T} \tau^{3}, \quad\left\|\mathcal{R}_{2}\left(\tau, t_{n}\right)\right\|_{a_{0}} \leq M_{T} \tau^{1+\epsilon}, \quad 0 \leq n \tau \leq T
$$

for some $\epsilon>0$.

Proof. Within the proof $M_{T}$ will again stand for a harmless number that depends only on $C_{T}$ which changes from line to line. By using Corollary 18, we can write

$$
\mathcal{R}_{2}\left(\tau, t_{n}\right)=\sum_{j=1}^{6} \mathcal{E}_{j}\left(\tau, t_{n}\right)
$$

where in the expansion of the right hand side given by (76), we define $\mathcal{E}_{1}\left(\tau, t_{n}\right)$ as the first 3 lines and then each line corresponds to one term. The estimates of $\mathcal{E}_{j}$ for $j \geq 2$ is a direct consequence of Assumption 2.3 and Assumption 1.1, we thus get

$$
\left\|\mathcal{E}_{j}\right\|_{\alpha_{0}} \leq M_{T}, \quad j \geq 2
$$

It thus only remains to estimate $\mathcal{E}_{1}\left(\tau, t_{n}\right)$. By using $(24)$, we first observe that

$$
\begin{aligned}
\mathcal{E}_{1}\left(\tau, t_{n}\right)=\int_{0}^{\tau} e^{(\tau-\xi) \mathcal{L}}\left[f\left(e^{\xi \mathcal{L}} u\left(t_{n}\right)+\xi f^{n}, e^{\xi \overline{\mathcal{L}}} \bar{u}\left(t_{n}\right)+\xi \overline{f^{n}}\right)-f\left(e^{\xi \mathcal{L}} u\left(t_{n}\right), e^{\xi \overline{\mathcal{L}}} \bar{u}\left(t_{n}\right)\right)\right] d \xi \\
-e^{\tau \mathcal{L}} \int_{0}^{\tau} \xi\left(D_{1} f^{n} \cdot f^{n}+D_{2} f^{n} \cdot \overline{f^{n}}\right) d \xi+\mathcal{E}_{1,1}\left(\tau, t_{n}\right),
\end{aligned}
$$

where

$$
\left\|\mathcal{E}_{1,1}\left(\tau, t_{n}\right)\right\|_{\alpha_{0}} \leq \tau C_{\alpha_{0}}\left(M_{T}, \sup _{(t, \xi) \in[0, T] \times[0, \tau]}\left\|\mathcal{R}_{2, a}(t, \xi)\right\|_{a_{0}}\right) \sup _{(t, \xi) \in[0, T] \times[0, \tau]}\left\|\mathcal{R}_{2, a}(t, \xi)\right\|_{a_{0}} .
$$

Then, by using only the first estimate of (4) for $\alpha=a_{0}$ and the expression (77), we get that

$$
\sup _{(t, \xi) \in[0, T] \times[0, \tau]}\left\|\mathcal{R}_{2, a}(t, \xi)\right\|_{a_{0}} \leq \tau C_{\alpha_{0}}\left(\sup _{[0, T]}\|u\|_{\alpha_{2}}\right) \leq \tau M_{T} .
$$


To estimate $\left\|\mathcal{R}_{2, a}(t, \xi)\right\|_{\alpha_{0}}$, we observe that thanks to the fundamental Theorem of calculus and the equation, we have

$$
\begin{aligned}
& \mathcal{R}_{2, a}(t, \xi) \\
& =\int_{0}^{\xi} \int_{0}^{s} e^{\left(\xi-s_{1}\right) \mathcal{L}}\left(-\mathcal{L}(f(u, \bar{u}))+D_{1} f(u, \bar{u}) \cdot(\mathcal{L} u+f(u, \bar{u}))+D_{2} f(u, \bar{u}) \cdot(\overline{\mathcal{L} u}+\bar{f}(u, \bar{u}))\right) d s_{1} d s \\
& \quad+\xi^{2} \varphi_{1}(\xi \mathcal{L}) \mathcal{L} f(u(t), \overline{u(t)})
\end{aligned}
$$

where in the above integral $u$ and $\bar{u}$ are always evaluated at the time $t+s_{1}$. Therefore, by using the assumption (25) and (79), we get that

$$
\sup _{(t, \xi) \in[0, T] \times[0, \tau]}\left\|\mathcal{R}_{2, a}(t, \xi)\right\|_{\alpha_{0}} \leq \tau^{2} M_{T}
$$

This finally implies thanks to (81) that

$$
\left\|\mathcal{E}_{1,1}\left(\tau, t_{n}\right)\right\|_{\alpha_{0}} \leq M_{T} \tau^{3}
$$

To estimate the remaining terms in $\mathcal{E}_{1}$, we can write

$$
\begin{aligned}
\mathcal{E}_{1}\left(\tau, t_{n}\right)=\int_{0}^{\tau} \xi e^{(\tau-\xi) \mathcal{L}} \int_{0}^{1}\left(D_{1} f\left(e^{\xi \mathcal{L}} u+s \xi f^{n}, e^{\xi \overline{\mathcal{L}}} \bar{u}+s \xi \overline{f^{n}}\right) \cdot f^{n}\right. & \\
& \left.+D_{2} f\left(e^{\xi \mathcal{L}} u+s \xi f^{n}, e^{\xi \overline{\mathcal{L}}} \bar{u}+s \xi \overline{f^{n}}\right) \cdot \overline{f^{n}}\right) d s d \xi \\
& -e^{\tau \mathcal{L}} \int_{0}^{\tau} \xi\left(D_{1} f^{n} \cdot f^{n}+D_{2} f^{n} \cdot \overline{f^{n}}\right) d \xi+\mathcal{E}_{1,1}\left(\tau, t_{n}\right)
\end{aligned}
$$

where $u$ and $\bar{u}$ are evaluated at $t_{n}$ and finally

$$
\begin{aligned}
& \mathcal{E}_{1}\left(\tau, t_{n}\right)=\int_{0}^{\tau} \int_{0}^{1} \xi \int_{0}^{\xi} e^{\left(\tau-s_{1}\right) \mathcal{L}}\left[-\mathcal{L}\left(D_{1} f\left(V_{s, s_{1}}, \overline{V_{s, s_{1}}}\right) \cdot f^{n}+D_{2} f\left(V_{s, s_{1}}, \overline{V_{s, s_{1}}}\right) \cdot \overline{f^{n}}\right)\right. \\
& +D_{1}^{2} f\left(V_{s, s_{1}}, \overline{V_{s, s_{1}}}\right) \cdot\left(e^{s_{1} \mathcal{L}} \mathcal{L} u_{n}+s f^{n}, f^{n}\right)+D_{12}^{2} f\left(V_{s, s_{1}}, \overline{V_{s, s_{1}}}\right) \cdot\left(e^{s_{1} \overline{\mathcal{L}}} \overline{\mathcal{L}} \overline{u_{n}}+s \overline{f^{n}}, f^{n}\right) \\
& +D_{12}^{2} f\left(V_{s, s_{1}}, \overline{V_{s, s_{1}}}\right) \cdot\left(e^{s_{1} \mathcal{L}} \mathcal{L} u_{n}+s f^{n}, \overline{f^{n}}\right)+D_{2}^{2} f\left(V_{s, s_{1}}, \overline{V_{s, s_{1}}}\right) \cdot\left(e^{s_{1}} \overline{\mathcal{L}} \overline{\mathcal{L}} \overline{u_{n}}+s \overline{f^{n}}, \overline{f^{n}}\right] d s_{1} d s d \xi \\
& +\mathcal{E}_{1,1}\left(\tau, t_{n}\right)
\end{aligned}
$$

where $V_{s, s_{1}}\left(t_{n}\right)=e^{s_{1} \mathcal{L}_{u}}\left(t_{n}\right)+s s_{1} f^{n}$. Consequently, by using (26) and (25) and (83), we get that

$$
\left\|\mathcal{E}_{1}\left(\tau, t_{n}\right)\right\|_{\alpha_{0}} \leq M_{T} \tau^{3}
$$

and hence finally the first part of (80).

It remains to estimate $\left\|\mathcal{R}_{2}\left(\tau, t_{n}\right)\right\|_{a_{0}}$. We use again (78) and the definition of the numerical flux in (19), to get

$$
\mathcal{R}_{2}\left(\tau, t_{n}\right)=u\left(t_{n+1}\right)-e^{\tau \mathcal{L}} u\left(t_{n}\right)-\tau e^{\tau \mathcal{L}} \Psi_{\text {num }, 2}^{\tau}\left(u\left(t_{n}\right)\right) .
$$

By using the Duhamel formula and the assumption (27), we easily get that

$$
\left\|\mathcal{R}_{2}\left(\tau, t_{n}\right)\right\|_{a_{0}} \leq M_{T} \tau .
$$

We finally get (80) from the same interpolation argument as in the study of the first-order case. 
4.2. Proof of Theorem 3. We follow the same lines as in the convergence proof of our first-order scheme. Let us set $e^{n}=u\left(t_{n}\right)-u^{n}, u^{n}$ being the numerical solution computed with (19). We get that

$$
e^{n+1}=e^{\tau \mathcal{L}}\left[e^{n}+\tau\left(\Psi_{\text {num }, 2}^{\tau}\left(u\left(t_{n}\right)\right)-\Psi_{\text {num }, 2}^{\tau}\left(u^{n}\right)\right)\right]+\mathcal{R}_{2}\left(\tau, t_{n}\right)
$$

By using (28) and Proposition 19, we get that for $0 \leq n \tau \leq T$,

$$
\left\|e^{n+1}\right\|_{\alpha_{0}} \leq\left\|e^{n}\right\|_{\alpha_{0}}+\tau C_{\alpha_{0}}\left(\left\|u\left(t_{n}\right)\right\|_{a_{0}},\left\|e^{n}\right\|_{a_{0}}\right)\left\|e^{n}\right\|_{\alpha_{0}}+M_{T} \tau^{3}
$$

In a similar way, by using (29), and Proposition 19, we obtain that

$$
\left\|e^{n+1}\right\|_{a_{0}} \leq\left\|e^{n}\right\|_{a_{0}}+\tau C_{a_{0}}\left(\left\|u\left(t_{n}\right)\right\|_{a_{0}},\left\|e^{n}\right\|_{a_{0}}\right)\left\|e^{n}\right\|_{a_{0}}+M_{T} \tau^{1+\epsilon} .
$$

We then easily get by induction that for $\tau$ sufficiently small, we have that

$$
\sup _{0 \leq n \leq N}\left\|e^{n}\right\|_{a_{0}}<+\infty, \quad \sup _{0 \leq n \leq N}\left\|e^{n}\right\|_{\alpha_{0}} \leq M_{T} \tau^{2} .
$$

This ends the proof.

\section{EXAMPLES}

5.1. Heat, NLS and Ginzburg-Landau type equations. To illustrate our general theory, we shall consider the equation

$$
\partial_{t} u-D \Delta u=\gamma u\left(1-|u|^{2}\right), \quad u_{/ t=0}=u_{0}
$$

where $u \in \mathbb{C}$, and $D, \gamma \in \mathcal{C}$ with $\operatorname{Re} D \geq 0$ so that nonlinear Schrödinger (NLS) type equations and nonlinear heat equations are included in the same model. We assume that the problem is set for $x \in \Omega \subset \mathbb{R}^{d}, d \leq 3$, where $\Omega$ is a smooth open set with compact boundary and we add the Dirichlet boundary condition

$$
u(t, \cdot) / \partial \Omega=0 .
$$

We thus set $X=L^{2}(\Omega)$ and $\mathcal{L}=D \Delta$ with domain $D(\mathcal{L})=H^{2} \cap H_{0}^{1}$. By standard characterization, we have that $X^{\alpha}=H^{2 \alpha} \cap H_{0}^{1}$ for $1 / 2 \leq \alpha \leq 1$ while for $0 \leq \alpha<1 / 2, X^{\alpha}=H^{2 \alpha}$.

Assumption 1.1 is then matched. The "nonlinearity" is given by

$$
f(u, \bar{u})=\gamma u-\gamma u^{2} \bar{u}
$$

so that $\mathcal{B}=I d$.

We shall check the assumptions for first-order convergence, we fix $a_{0}$ any number strictly bigger than $d / 4$ arbitrarily close to it so that $H^{2 a_{0}}(\Omega) \subset L^{\infty}$ and $a_{1}=1$ (the propagation of higher order regularity would require additional compatibility conditions for the initial data).

The estimate (4) then follows by standard Moser-Gagliardo-Nirenberg-Sobolev estimates since for every $\alpha \geq 0$

$$
\left\||u|^{2} u\right\|_{H^{2 \alpha}} \lesssim\|u\|_{H^{2 a_{0}}}^{2}\|u\|_{H^{2 \alpha}}, \quad 2 a_{0}>d / 2 .
$$

Let us consider the scheme (6) for this model and measure the error in $L^{2}$ so that $\alpha_{0}=0$.

Then, we have the explicit expression

$$
\mathcal{C}[f, \mathcal{L}](v, w)=\gamma D\left(\Delta\left(v^{2} w\right)-2 v \Delta v w-v^{2} \Delta w\right)=\gamma D(4 v \nabla v \cdot \nabla w+2 \nabla v \cdot \nabla v w) .
$$

We thus deduce that

$$
\|\mathcal{C}[f, \mathcal{L}](v, w)\|_{L^{2}} \lesssim\left(\|v\|_{L^{\infty}}+\|w\|_{L^{\infty}}\right)\left(\|\nabla v\|_{L^{4}}\|\nabla w\|_{L^{4}}+\|\nabla v\|_{L^{4}}^{2}\right) .
$$

Since $H^{2 a_{0}} \subset L^{\infty}$, and $H^{s} \subset L^{4}$ with $s=d / 4$, we can take

$$
\alpha_{1}=\frac{1}{2}\left(1+\frac{d}{4}\right)
$$

(note that we can always assume that $d / 2<2 a_{0}<1+d / 4$ when $d \leq 3$.) 
Next, we have that $F(u) \cdot e^{s \mathcal{A}} \mathcal{C}[G, \mathcal{A}](w)=0$, since $f$ is linear in $\bar{u}$. Therefore (11) is also matched.

It remains to check Assumption (2.2) (recall that we take $\alpha_{0}=0$ here).

- The first part of (12) is again a consequence of $H^{2 a_{0}}(\Omega) \subset L^{\infty}$ For the second part, we use

$$
\left\||u|^{2} u\right\|_{L^{2}} \leq\|u\|_{L^{\infty}}\|u\|_{L^{4}}^{2}
$$

and the estimate follows from the embedding $H^{\frac{d}{4}} \subset L^{4}$.

- In this specific case, we have

$$
\mathcal{B}\left((F(u)) \cdot \varphi_{1}(\tau, \mathcal{A}) G(w)\right)=\gamma u-\gamma u^{2} \varphi_{1}(\tau, \mathcal{A}) w
$$

since $\varphi_{1}(\tau, \mathcal{A})$ is bounded on Sobolev spaces, the estimates (13), (14) and (15) then also follow from the above arguments.

We then obtain from Theorem 2, that:

Corollary 20. For $1 \leq d \leq 3$ and for every $u_{0} \in H^{1+\frac{d}{4}}(\Omega) \cap H_{0}^{1}(\Omega)$, let $T>0$ and $u \in$ $\mathcal{C}\left([0, T], H^{1+\frac{d}{4}}(\Omega) \cap H_{0}^{1}(\Omega)\right)$ the unique solution of (84) given by Theorem 1 , let $u^{n}$ denote the numerical solution given by (6). Then there exists $C_{T}>0$ such that

$$
\left\|u(n \tau)-u^{n}\right\|_{L^{2}} \leq C_{T} \tau, \quad 0 \leq n \tau \leq T .
$$

Note that we require only $1+d / 4$ derivatives to get first-order convergence in contrast to classical schemes which require (at least) 2 derivatives. The same result holds for periodic boundary conditions. Corollary 20 does not use any smoothing properties of the PDE, it could be improved by using parabolic smoothing if $\operatorname{Re} D>0$. It could also be improved when $\operatorname{Re} D=0$ if $\Omega$ is nice (non-trapping exterior domain), or if $\Omega=\mathbb{T}^{d}$ by using tools from dispersive PDE in the convergence analysis of the scheme (e.g. discrete Bourgain-space type estimates) see [23], [24].

Also note that for periodic boundary conditions $\left(x \in \mathbb{T}^{d}\right)$ or the full space $\left(x \in \mathbb{R}^{d}\right)$ if we measure the error in high order Sobolev spaces $H^{r}$ with $r=2 \alpha_{0}>d / 2$ we obtain the global error estimate $\left\|u(n \tau)-u^{n}\right\|_{r} \leq C_{T} \tau$ for solutions in $H^{r+1}$.

Remark 21. In the special case of the periodic Schrödinger equation with polynomial nonlinearities $u^{p} \bar{u}^{m}$ the second-order Duhamel integrator (19) improves previous methods ([15]). For periodic boundary conditions it is similar to the second-order scheme developed in [5] based on the combination of a resonance based discretisation (which is so far restricted to periodic boundary conditions) coupled with suitable filter functions. For the favorable error behaviour at low regularity, see in particular [5, Fig. 3].

Remark 22. In case of the cubic Schrödinger equation the filtered Lie splitting (18) takes the form

$$
\left.u^{\ell+1}=e^{\tau \mathcal{L}} \mathrm{e}^{\tau u^{\ell}\left(\Psi(\tau) \overline{u^{\ell}}\right.}\right) u^{\ell} \quad \text { with } \quad \Psi(\tau)=\varphi_{1}(-2 i \tau \Delta) .
$$

In contrast to its unfiltered counterpart $(\Psi(\tau) \equiv 1)$, the filtered Lie splitting $(87)$ only involves one additional derivative of the solution in the local error (i.e, $\nabla u$ ) instead of two (i.e., $\Delta u$ ). This can be seen by reformulating the filtered Lie splitting as follows

$$
u^{\ell+1}=e^{\tau \mathcal{L}}\left[\left(1+\tau u^{\ell}\left(\Psi(\tau) \overline{u^{\ell}}\right)\right) u^{\ell}\right]+\mathcal{R}\left(\tau, u^{\ell}\right)
$$

where the first term corresponds to the low regularity scheme (6), which only involves first order derivatives in the local error, see (86), and the remainder

$$
\mathcal{R}\left(\tau, u^{\ell}\right)=e^{\tau \mathcal{L}}\left[\left(\mathrm{e}^{\tau u^{\ell}\left(\Psi(\tau) \overline{u^{\ell}}\right)}-1-\tau u^{\ell}\left(\Psi(\tau) \overline{u^{\ell}}\right)\right) u^{\ell}\right]
$$

is of order $\mathcal{O}\left(\tau^{2} q\left(u^{\ell}\right)\right)$ for some polynomial $q$. 
5.2. Half-waves. Let us consider the half-wave equation

$$
i \partial_{t} u+|\nabla| u= \pm|u|^{2} u, \mathcal{L}=i|\nabla|, \quad u_{/ t=0}=u_{0}
$$

In this situation the low regularity scheme at first-order takes the form (cf. (6))

$$
u^{\ell+1}=e^{i \tau|\nabla|}\left(u^{\ell} \mp i \tau\left(u^{\ell}\right)^{2} \varphi_{1}(-2 i \tau|\nabla|) \overline{u^{\ell}}\right)
$$

and at second-order it reads (cf. (19))

$$
\begin{aligned}
u^{\ell+1} & =e^{i \tau|\nabla|} u^{\ell} \mp i \tau e^{i \tau|\nabla|}\left(\left(u^{\ell}\right)^{2} \varphi_{1}(-2 i \tau|\nabla|) \bar{u}^{\ell}\right) \mp i \tau\left(e^{i \tau|\nabla|} u^{\ell}\right)^{2} \varphi_{2}(-2 i \tau|\nabla|) e^{i \tau|\nabla|} \bar{u}^{\ell} \\
& \pm i \tau e^{i \tau|\nabla|}\left(\left(u^{\ell}\right)^{2} \varphi_{2}(-2 i \tau|\nabla|) \bar{u}^{\ell}\right)-\frac{\tau^{2}}{2} e^{i \tau|\nabla|}\left(\left|u^{\ell}\right|^{4} u^{\ell}\right) .
\end{aligned}
$$

In the following we analyze the order of convergence of the above schemes.

5.2.1. First-order scheme. We shall first analyze our first-order scheme in dimension 1 with periodic boundary conditions thus for $x \in \mathbb{T}, d=1$. Here, we have

$$
f(u, \bar{u})=\mp i u^{2} \bar{u}
$$

so that again, $\mathcal{B}=I d$.

We set $X=L^{2}(\mathbb{T}), \mathcal{L}=i|\nabla|$ with domain $D(\mathcal{L})=H^{1}(\mathbb{T})$. We thus have that $X^{\alpha}=H^{\alpha}(\mathbb{T})$. Assumption 1.1 is then matched. We fix now $a_{0}$ any number strictly bigger than $1 / 2$ arbitrarily close to it, and take $a_{1}=\alpha_{1}=3 / 4$ and we will again measure the error in $L^{2}$ so that $\alpha_{0}=0$. Assumptions 1.2, 2.2 are matched thanks to the same arguments as above. Then, we have that

$$
\mathcal{C}[f, \mathcal{L}](v, w)= \pm i\left(|\nabla|\left(v^{2} w\right)-|\nabla|\left(v^{2}\right) w-v^{2}|\nabla| w+\left(|\nabla|\left(v^{2}\right)-2 v|\nabla v|\right) w\right) .
$$

To estimate this term, we shall use the following estimate

$$
\||\nabla|(f g)-|\nabla| f g-f|\nabla| g\|_{L^{2}} \lesssim\|f\|_{H^{\frac{1}{2}+\frac{d}{4}}}\|g\|_{H^{\frac{1}{2}+\frac{d}{4}}}
$$

which can be obtained as a consequence of some recent version of the generalized Leibnitz rule [18]. We shall provide a direct proof of the above estimate which is valid for the torus in Section 6 . The latter in particular implies that

$$
\begin{aligned}
& \|\mathcal{C}[f, \mathcal{L}](v, w)\|_{L^{2}} \lesssim\left\|v^{2}\right\|_{H^{\frac{1}{2}+\frac{d}{4}}}\|w\|_{H^{\frac{1}{2}+\frac{d}{4}}}+\|w\|_{L^{\infty}}\left\|\nabla\left|\left(v^{2}\right)-2 v\right| \nabla v \mid\right\|_{L^{2}} \\
& \lesssim\|v\|_{L^{\infty}}\|v\|_{H^{\frac{1}{2}+\frac{d}{4}}}\|w\|_{H^{\frac{1}{2}+\frac{d}{4}}}+\|w\|_{L^{\infty}}\|v\|_{H^{\frac{1}{2}+\frac{d}{4}}}^{2} .
\end{aligned}
$$

Therefore, with $\alpha_{1}=3 / 4$, we get

$$
\mid \mathcal{C}[f, \mathcal{L}](v, w)\left\|_{L^{2}} \lesssim\right\| w\left\|_{H^{\alpha_{1}}}\right\| v \|_{H^{\alpha_{1}}}^{2} .
$$

Next, we have that $F(u) \cdot e^{s \mathcal{A}} \mathcal{C}[G, \mathcal{A}](w)=0$, since $f$ is linear in $\bar{u}$ therefore (11) is also matched. We can check (4) and Assumption 2.2 as in the previous example.

We then obtain from Theorem 2 , that

Corollary 23. For for every $u_{0} \in H^{\frac{3}{4}}(\mathbb{T})$. Let $T>0$ and $u \in \mathcal{C}\left([0, T], H^{\frac{3}{4}}(\mathbb{T})\right.$ the unique solution of (84) given by Theorem 1, let $u^{n}$ denote the numerical solution given by (6). Then there exists $C_{T}>0$ such that

$$
\left\|u(n \tau)-u^{n}\right\|_{L^{2}} \leq C_{T} \tau, \quad 0 \leq n \tau \leq T .
$$

Note that classical schemes would require initial data (at least) in $H^{1}$.

In higher dimensions, 2, 3 for example, at first-order our convergence result does not improve on the classical regularity assumption. The main reason is that the order of the operator $\mathcal{L}$, which is 1 in this case, is smaller than $d / 2$ which is the minimal $a_{0}$ that we can take to get a local Cauchy Theory without using more sophisticated harmonic analysis tools. In this case, the estimate for 
the commutator $\mathcal{C}[f, \mathcal{L}]$ is not better than the estimate of each of the terms alone. We could improve the first-order error estimate by adapting the harmonic analysis tools developed in [24]. Nevertheless, even without any sophisticated tools our second-order convergence result yields a significant improvement as we shall see below.

5.2.2. Second-order scheme. To illustrate our second-order theory, we shall now consider (88) for $d=2$ still with periodic boundary conditions i.e $x \in \mathbb{T}^{2}$. We still have $X^{\alpha}=H^{\alpha}\left(\mathbb{T}^{d}\right)$. We choose again to measure the error in $L^{2}$ so $\alpha_{0}=0$ and we fix $a_{0}>d / 2$ arbitrarily close to it. We first check the estimate $(21)$ to fix $\alpha_{2}$. Note that again $(22)$ and (23) are trivially satisfied, $\mathcal{C}[G, \mathcal{A}]=0$ since $G$ is linear. Let us set $T\left(v_{1}, v_{2}, v_{3}\right)=v_{1} v_{2} v_{3}$. We shall estimate $\left\|\mathcal{C}^{2}[T, \mathcal{L}]\left(v_{1}, v_{2}, v_{3}\right)\right\|_{L^{2}}$. The estimate of $\left\|\mathcal{C}^{2}[f, \mathcal{L}](v, w)\right\|_{L^{2}}$ then follows by observing that $f(v, w)=T(v, v, w)$. By expanding $\mathcal{C}^{2}[T, \mathcal{L}]\left(v_{1}, v_{2}, v_{3}\right)$, we get that

$$
\mathcal{C}^{2}[T, \mathcal{L}]\left(v_{1}, v_{2}, v_{3}\right)=|\nabla|^{2}\left(v_{1} v_{2} v_{3}\right)-|\nabla|^{2} v_{1} v_{2} v_{3}-v_{1}|\nabla|^{2} v_{2} v_{3}-v_{1} v_{2}|\nabla|^{2} v_{3}+\mathcal{R}_{1}+\mathcal{R}_{2}
$$

where

$$
\mathcal{R}_{1}=\sum_{i, j, k} v_{i}|\nabla| v_{j}|\nabla| v_{k}, \quad \mathcal{R}_{2}=\sum_{i, j, k}\left(|\nabla|\left(v_{i} v_{j}|\nabla| v_{k}\right)-v_{i} v_{j}|\nabla|^{2} v_{k}\right) .
$$

The sums are finite sums, we do not need to explicit them. We can easily estimate $\mathcal{R}_{1}$ by using the Hölder inequality and the Sobolev embedding $H^{\frac{1}{2}} \subset L^{4}$ in dimension 2. We get

$$
\left\|\mathcal{R}_{1}\right\|_{L^{2}} \lesssim \sum_{i, j, k}\left\|v_{i}\right\|_{L^{\infty}}\left\||\nabla| v_{j}\right\|_{L^{4}}\left\||\nabla| v_{k}\right\|_{L^{4}} \lesssim \sum_{i, j, k}\left\|v_{i}\right\|_{H^{a_{0}}}\left\|v_{j}\right\|_{H^{\frac{3}{2}}}\left\|v_{k}\right\|_{H^{\frac{3}{2}}} .
$$

For $\mathcal{R}_{2}$ we use the Kato-Ponce commutator estimate (see for example [18] Theorem 1.9) to get

$$
\left\|\mathcal{R}_{2}\right\|_{L^{2}} \lesssim \sum_{i, j, k}\left\|\nabla\left(v_{i} v_{j}\right)\right\|_{L^{4}}\left\||\nabla| v_{k}\right\|_{L^{4}} \lesssim \sum_{i, j, k}\left\|v_{i}\right\|_{L^{\infty}}\left\|v_{j}\right\|_{H^{\frac{3}{2}}}\left\|v_{k}\right\|_{H^{\frac{3}{2}}} .
$$

It remains to estimate the leading term in (89). Since $|\nabla|^{2}=-\Delta$ we can expand with the classical Leibnitz formula. We obtain

$$
\mathcal{C}^{2}[T, \mathcal{L}]\left(v_{1}, v_{2}, v_{3}\right)=-\sum_{i, j, k} v_{i} \nabla v_{j} \cdot \nabla v_{k}+\mathcal{R}_{1}+\mathcal{R}_{2}
$$

Therefore, from the same estimates as previously, we obtain that

$$
\left\|\mathcal{C}^{2}[T, \mathcal{L}]\left(v_{1}, v_{2}, v_{3}\right)\right\| \lesssim\left\|v_{1}\right\|_{H^{\frac{3}{2}}}\left\|v_{2}\right\|_{H^{\frac{3}{2}}}\left\|v_{3}\right\|_{H^{\frac{3}{2}}} \cdot
$$

We thus choose $\alpha_{2}=\frac{3}{2}$. The other assumptions are then again a consequence of the tame estimate, for $s \geq 0$ :

$$
\|u v w\|_{H^{s}} \lesssim\|u\|_{H^{a_{0}}}\|v\|_{H^{a_{0}}}\|w\|_{H^{s}}+\|v\|_{H^{a_{0}}}\|w\|_{H^{a_{0}}}\|u\|_{H^{s}}+\|w\|_{H^{a_{0}}}\|u\|_{H^{a_{0}}}\|v\|_{H^{s}} .
$$

We thus obtain:

Corollary 24. For every $u_{0} \in H^{\frac{3}{2}}\left(\mathbb{T}^{2}\right)$. Let $T>0$ and $u \in \mathcal{C}\left([0, T], H^{\frac{3}{2}}\left(\mathbb{T}^{2}\right)\right)$ the unique solution of (88) given by Theorem 1, let $u^{n}$ denote the numerical solution given by (19). Then there exists $C_{T}>0$ such that

$$
\left\|u(n \tau)-u^{n}\right\|_{L^{2}} \leq C_{T} \tau^{2}, \quad 0 \leq n \tau \leq T .
$$

Note that second-order convergence of classical schemes for the Half-wave equation (88) requires (at least) $H^{2}$ solutions. 
5.3. Wave and Klein-Gordon type equations. Our general framework can also be applied to Klein-Gordon and wave equations: Let us for instance consider the nonlinear Klein-Gordon equation

$$
\partial_{t t} z-\Delta z+m^{2} z=g(z), \quad z(0)=u_{0}, \quad \partial_{t} z(0)=u_{1},
$$

where for simplicity we assume non zero mass $m \neq 0$ and real-valued solutions $z(t, x) \in \mathbb{R}$. However, we may also deal with the complex setting $z(t, x) \in \mathbb{C}$ and wave equations $m=0$.

In a first step let us rewrite the Klein-Gordon equation (90) as a first-order complex system. We consider that $x \in \mathbb{T}^{d}$ for $d \leq 3$ so that

$$
\langle\nabla\rangle_{m}=\sqrt{-\Delta+m^{2}}
$$

can be defined with Fourier series. We get

$$
i \partial_{t} u=-\langle\nabla\rangle_{m} u+\langle\nabla\rangle_{m}^{-1} g\left(\left(\frac{1}{2}(u+\bar{u})\right) .\right.
$$

The latter is obtained via the classical transformation

$$
u=z-i\langle\nabla\rangle_{m}^{-1} \partial_{t} z
$$

where

$$
\langle\nabla\rangle_{m}=\sqrt{-\Delta+m^{2}}
$$

is an invertible operator (recall that $m \neq 0$ ) and

$$
z=\frac{1}{2}(u+\bar{u})=\operatorname{Re}(u)
$$

We thus define $\mathcal{L}=i\langle\nabla\rangle_{m}$, with $X=L^{2}\left(\mathbb{T}^{d}\right), D(\mathcal{L})=H^{1}\left(\mathbb{T}^{d}\right)$ and hence $X^{\alpha}=H^{\alpha}\left(\mathbb{T}^{d}\right)$. Assumption 1.1 is clearly satisfied.

To illustrate our theory, we shall consider two classical nonlinearities:

- power type: $g(z)=z^{2}$

- Sine-Gordon: $g(z)=-\sin z$.

We have

$$
f(u, \bar{u})=-i\langle\nabla\rangle_{m}^{-1} g\left(\left(\frac{1}{2}(u+\bar{u})\right)\right.
$$

so that $f$ is under the form (3) with

$$
\begin{aligned}
& f(v, w)=\mathcal{B}(F(v) \cdot G(w))=-\frac{1}{4} i\langle\nabla\rangle_{m}^{-1}\left(v^{2}+w^{2}+2 v w\right), \quad g(z)=z^{2}, \\
& f(v, w)=\mathcal{B}(F(v) \cdot G(w))=i\langle\nabla\rangle_{m}^{-1}\left(\sin \left(\frac{1}{2} v\right) \cos \left(\frac{1}{2} w\right)+\cos \left(\frac{1}{2} v\right) \sin \left(\frac{1}{2} w\right)\right), \quad g(z)=-\sin z .
\end{aligned}
$$

Note that in this case, $\mathcal{B}$ is non-trivial, we have, $\mathcal{B}=\langle\nabla\rangle_{m}^{-1}$. We can thus use our schemes for this formulation. A natural space to measure the error for wave type equations in terms of $\left(z, \partial_{t} z\right)$ is $H^{1} \times L^{2}$. In terms of $u$ with the definition (92) the $H^{1}$ norm is hence a natural choice. We shall thus choose $\alpha_{0}=1$.

5.3.1. First-order scheme. We shall study our first-order-scheme (6), in dimension $1, d=1$. Let us then check our assumptions 1.2, 2.1, 2.2 with $\alpha_{0}=1$.

Let us start with the nonlinearity given by (93). We can take $a_{0}=0$ and we we can take $a_{1}=\alpha_{1}=\frac{1}{2}+\frac{1}{4}=\frac{3}{4}$. Indeed, let us first check Assumption 2.1. We use again the following inequality

$$
\begin{aligned}
\|\langle\nabla\rangle_{m}^{-1}\left(\langle\nabla\rangle_{m}(v w)-\langle\nabla\rangle_{m} v w-\right. & \left.v\langle\nabla\rangle_{m} w\right) \|_{H^{1}} \\
& \left.\lesssim \|\langle\nabla\rangle_{m}(v w)-\langle\nabla\rangle_{m} v w-v\langle\nabla\rangle_{m} w\right)\left\|_{L^{2}} \lesssim\right\| v\left\|_{H^{\alpha_{1}}}\right\| w \|_{H^{\alpha_{1}}}
\end{aligned}
$$


where we have used Lemma 27 (see below) for the last inequality together with the Sobolev embedding $H^{\frac{d}{4}} \subset L^{4}$. The estimate (95) implies (10), (11) and motivates the choice of $\alpha_{1}$. Let us now check the other assumptions. We have by duality (since $H^{1} \subset L^{\infty}$ ) that

$$
\left\|\langle\nabla\rangle_{m}^{-1}(v w)\right\|_{L^{2}} \lesssim\|v w\|_{H^{-1}} \lesssim\|v w\|_{L^{1}} \lesssim\|v\|_{L^{2}}\|w\|_{L^{2}}
$$

and

$$
\|f(u, \bar{u})\|_{H^{\frac{3}{4}}} \lesssim\left\||u|^{2}\right\|_{H^{-\frac{1}{4}}} \lesssim\left\||u|^{2}\right\|_{L^{2}} \lesssim\|u\|_{L^{2}}\|u\|_{L^{\infty}} \lesssim\|u\|_{L^{2}}\|u\|_{H^{\frac{3}{4}}} \cdot
$$

This yields (4) (the case $\alpha \in(0,3 / 4]$ can be obtained from similar arguments and is actually not needed) and (15). The estimates (14), (12), (13) are a consequence of the following estimate: for $\alpha \leq 1$

$$
\left\|\langle\nabla\rangle_{m}^{-1}(v w)\right\|_{H^{\alpha}} \lesssim\|v w\|_{L^{2}} \lesssim\|v\|_{L^{2}}\|w\|_{L^{\infty}} \lesssim\|v\|_{L^{2}}\|w\|_{H^{\frac{3}{4}}}
$$

The nonlinearity (94) can be handled in a similar way, the only new non-trivial estimates to obtain are (10), (11). We first observe that

$$
\left.\|\langle\nabla\rangle_{m}^{-1}\left(\langle\nabla\rangle_{m} \sin u-\cos u\langle\nabla\rangle_{m} u\right)\right)\left\|_{H^{1}} \lesssim\right\|\langle\nabla\rangle_{m} \sin u-\cos u\langle\nabla\rangle_{m} u \|_{L^{2}}
$$

So that we only need to estimate $\left\|\langle\nabla\rangle_{m} \sin u-\cos u\langle\nabla\rangle_{m} u\right\|_{L^{2}}$. Details will be given below in Lemma 28. We obtain again that

$$
\left\|\langle\nabla\rangle_{m} \sin u-\cos u\langle\nabla\rangle_{m} u\right\|_{L^{2}} \lesssim C\left(\|u\|_{H^{\frac{3}{4}}}\right) .
$$

We thus obtain that

Corollary 25. For for every $u_{0} \in H^{\frac{3}{4}}(\mathbb{T}), u_{1} \in H^{-\frac{1}{4}}(\mathbb{T})$ Let $T>0$ and $z \in \mathcal{C}\left([0, T], H^{\frac{3}{4}}(\mathbb{T})\right) \cap$ $\mathcal{C}^{1}\left([0, T], H^{-\frac{1}{4}}(\mathbb{T})\right)$ the unique solution of $(90)$, with $g(z)=z^{2}$ or $g(z)=\sin z$. Let $u^{n}$ denote the numerical solution given by (6). Then there exists $C_{T}>0$ such that

$$
\left\|z(n \tau)-\operatorname{Re} u^{n}\right\|_{H^{1}}+\left\|\partial_{t} z(n \tau)-\operatorname{Im} u^{n}\right\|_{L^{2}} \leq C_{T} \tau, \quad 0 \leq n \tau \leq T .
$$

Such an error estimate for a classical first-order scheme for the equation (90) would require (at least) $H^{1}$ solutions.

In higher dimensions, our second-order scheme will give more significant improvements.

5.3.2. Second-order scheme. We shall now study (90) formulated as (91) for $x \in \mathbb{T}^{3}$, and $g(z)=z^{2}$. We shall again measure the error in $H^{1}$ so that $\alpha_{0}=1$. In this case, we could take $a_{0}=1 / 2$, nevertheless, since we will be forced to take $\alpha_{2}=7 / 4$, which is now bigger than $\alpha_{0}$, it is convenient to choose $a_{0}=\alpha_{0}=1, a_{1}=\alpha_{2}=7 / 4$. To get (4), we observe that with our choice, by using the embedding $H^{\frac{3}{4}} \subset L^{4}$ in dimension 3 , we can write

$$
\left\|\langle\nabla\rangle_{m}^{-1}(v w)\right\|_{H^{1}} \lesssim\|v w\|_{L^{2}} \lesssim\|v\|_{L^{4}}\|w\|_{L^{4}} \lesssim\|v\|_{H^{\frac{3}{4}}}\|w\|_{H^{\frac{3}{4}}} .
$$

This yields (4) for $\alpha=a_{0}$. For $\alpha \in\left(a_{0}, a_{1}\right], a_{1}=\alpha_{1}=7 / 4$, we use that

$$
\left\|\langle\nabla\rangle_{m}^{-1}(v w)\right\|_{H^{a_{1}}} \lesssim\|v w\|_{H^{\frac{1}{4}}}
$$

and hence by using the generalized Leibnitz rule, and the embeddings $H^{1} \subset L^{6}, H^{\frac{1}{2}} \subset L^{3}$ we get

$$
\|v w\|_{H^{\frac{1}{4}}} \lesssim\left\|\langle\nabla\rangle^{\frac{1}{4}} v\right\|_{L^{6}}\|w\|_{L^{3}}+\left\|\langle\nabla\rangle^{\frac{1}{4}} w\right\|_{L^{6}}\|v\|_{L^{3}} \lesssim\|v\|_{H^{\frac{5}{4}}}\|w\|_{H^{\frac{1}{2}}}+\|w\|_{H^{\frac{5}{4}}}\|v\|_{H^{\frac{1}{2}}} \cdot
$$

We have thus obtained that

$$
\left\|\langle\nabla\rangle_{m}^{-1}(v w)\right\|_{H^{a_{1}}} \lesssim\|v\|_{H^{a_{0}}}\|w\|_{H^{a_{1}}}+\|w\|_{H^{a_{0}}}\|v\|_{H^{a_{1}}} .
$$

This yields (4). For (24), we use that

$$
\left\|\langle\nabla\rangle_{m}^{-1}(v w)\right\|_{H^{1}} \lesssim\|v w\|_{L^{2}} \lesssim\|v\|_{L^{3}}\|w\|_{L^{6}} \lesssim\|v\|_{H^{\frac{1}{2}}}\|w\|_{H^{1}},
$$


since $H^{1} \subset L^{6}, H^{\frac{1}{2}} \subset L^{3}$. For (25), we have that for $k=0,1$,

and that

$$
\begin{gathered}
\left\|\langle\nabla\rangle_{m}^{k}\left(\langle\nabla\rangle_{m}^{-1} u^{2}\right)\right\|_{H^{1}} \lesssim\|u \nabla u\|_{L^{2}} \lesssim\|u\|_{L^{4}}\|\nabla u\|_{L^{4}} \lesssim\|u\|_{H^{\frac{7}{4}}}^{2}, \\
\left\|\langle\nabla\rangle_{m}^{-1}\left(u\langle\nabla\rangle_{m} u\right)\right\|_{H^{1}} \lesssim\|u \nabla u\|_{L^{2}} \lesssim\|u\|_{H^{\frac{7}{4}}}^{2},
\end{gathered}
$$

$$
\begin{aligned}
&\left\|\langle\nabla\rangle_{m}^{-1}\left(u\langle\nabla\rangle_{m}^{-1}\left(v^{2}\right)\right)\right\|_{H^{1}}\|\lesssim\| u\langle\nabla\rangle_{m}^{-1} v^{2}\left\|_{L^{2}} \lesssim\right\| u\left\|_{L^{3}}\right\|\langle\nabla\rangle_{m}^{-1} v^{2} \|_{L^{6}} \\
& \lesssim\|u\|_{H^{\frac{1}{2}}}\left\|v^{2}\right\|_{L^{2}} \lesssim\|u\|_{H^{\frac{1}{2}}}\|v\|_{L^{4}}^{2} \lesssim\|u\|_{H^{\frac{1}{2}}}\|v\|_{H^{\frac{3}{4}}}^{2} .
\end{aligned}
$$

In a similar way, we get (26) from

$$
\|\langle\nabla\rangle_{m}\left(\langle\nabla\rangle_{m}^{-1}\left(u\langle\nabla\rangle_{m}^{-1}\left(v^{2}\right)\right)\left\|_{H^{1}} \lesssim\right\| u\left\|_{H^{1}}\right\|\langle\nabla\rangle_{m}^{-1}\left(v^{2}\right)\left\|_{L^{\infty}}+\right\| u\left\|_{L^{6}}\right\| v\left\|_{L^{6}}^{2} \lesssim\right\| u\left\|_{H^{1}}\right\| v \|_{H^{1}}^{2},\right.
$$

since $\alpha_{2}=7 / 4$.

To get (28) (29) (since we have chosen $a_{0}=\alpha_{0}=1$, there is only one set of assumptions to check) we can use again (96) and (98), so that it only remains to estimate the terms of the type $D_{i} f \cdot f$, we use that

$$
\|\langle\nabla\rangle_{m}^{-1}\left(u\langle\nabla\rangle_{m}^{-1}(v w)\left\|_{H^{1}} \lesssim\right\| u\left\|_{L^{3}}\right\|\langle\nabla\rangle_{m}^{-1}(v w)\left\|_{L^{6}} \lesssim\right\| u\left\|_{H^{\frac{1}{2}}}\right\| v w\left\|_{L^{2}} \lesssim\right\| u\left\|_{H^{1}}\right\| v\left\|_{H^{1}}\right\| w \|_{H^{1}} .\right.
$$

To get (28), we can use again (97) since $a_{1}=\alpha_{2}$ with the following estimate to handle the terms involving $D_{i} f \cdot f$

$$
\begin{aligned}
& \|\langle\nabla\rangle_{m}^{-1}\left(u\langle\nabla\rangle_{m}^{-1}(v w)\left\|_{H^{\frac{7}{4}}} \lesssim\right\| u\langle\nabla\rangle_{m}^{-1}(v w) \|_{H^{\frac{3}{4}}}\right. \\
& \quad \lesssim\left\|\langle\nabla\rangle_{m}^{\frac{3}{4}} u\right\|_{L^{3}}\left\|\langle\nabla\rangle_{m}^{-1}(v w)\right\|_{L^{6}}+\|u\|_{L^{6}}\|\|\langle\nabla\rangle_{m}^{-\frac{1}{4}}(v w)\left\|_{L^{3}} \lesssim\right\| u\left\|_{H^{\frac{5}{4}}}\right\| v\left\|_{H^{\frac{5}{4}}}\right\| w \|_{H^{\frac{5}{4}}} .
\end{aligned}
$$

It only remains to check Assumption 2.3. Let us set $B(v, w)=\langle\nabla\rangle_{m}^{-1}(v w)$, we can easily deduce Assumption 2.3, if we can prove that

$$
\left\|\mathcal{C}^{2}\left[B,\langle\nabla\rangle_{m}\right](v, w)\right\|_{H^{1}} \lesssim\|v\|_{H^{\frac{7}{4}}}\|w\|_{H^{\frac{7}{4}}} .
$$

By explicit computation we have

$$
\begin{aligned}
& \mathcal{C}^{2}\left[B,\langle\nabla\rangle_{m}\right](v, w)=\langle\nabla\rangle_{m}^{-1}\left(\langle\nabla\rangle_{m}^{2}(v w)-\langle\nabla\rangle_{m}^{2} v w-v\langle\nabla\rangle_{m}^{2} w\right) \\
& +2\langle\nabla\rangle_{m}^{-1}\left(\langle\nabla\rangle_{m} v\langle\nabla\rangle_{m} w\right)-2\langle\nabla\rangle_{m}^{-1}\left(\langle\nabla\rangle_{m}\left(v\langle\nabla\rangle_{m} w\right)-v\langle\nabla\rangle_{m}^{2} w+\langle\nabla\rangle_{m}\left(w\langle\nabla\rangle_{m} v\right)-w\langle\nabla\rangle_{m}^{2} v\right) .
\end{aligned}
$$

Since $\langle\nabla\rangle_{m}^{2}=m^{2}-\Delta$, we get from the standard Leibnitz formula that

$$
\begin{aligned}
\left\|\langle\nabla\rangle_{m}^{-1}\left(\langle\nabla\rangle_{m}^{2}(v w)-\langle\nabla\rangle_{m}^{2} v w-v\langle\nabla\rangle_{m}^{2} w\right)\right\|_{H^{1}} \lesssim \|\langle\nabla\rangle_{m}^{2}(v w) & -\langle\nabla\rangle_{m}^{2} v w-v\langle\nabla\rangle_{m}^{2} w \|_{L^{2}} \\
& \lesssim\|v\|_{L^{4}}\|w\|_{L^{4}}+\|\nabla v\|_{L^{4}}\|\nabla w\|_{L^{4}}
\end{aligned}
$$

and hence from the embedding $H^{\frac{3}{4}} \subset L^{4}$ in dimension 3 , we get

$$
\left\|\langle\nabla\rangle_{m}^{-1}\left(\langle\nabla\rangle_{m}^{2}(v w)-\langle\nabla\rangle_{m}^{2} v w-v\langle\nabla\rangle_{m}^{2} w\right)\right\|_{H^{1}} \lesssim\|v\|_{H^{\frac{7}{4}}}\|w\|_{H^{\frac{7}{4}}} \cdot
$$

For the second term in the right hand side of (100), we use again

$$
\left\|\langle\nabla\rangle_{m}^{-1}\left(\langle\nabla\rangle_{m} v\langle\nabla\rangle_{m} w\right)\right\|_{H^{1}} \lesssim\|v\|_{H^{\frac{7}{4}}}\|w\|_{H^{\frac{7}{4}}} \cdot
$$

The most difficult term is $\langle\nabla\rangle_{m}^{-1}\left(\langle\nabla\rangle_{m}\left(v\langle\nabla\rangle_{m} w\right)-v\langle\nabla\rangle_{m}^{2} w\right)$, for this one, we use again the KatoPonce inequality to write

$$
\begin{aligned}
&\left\|\langle\nabla\rangle_{m}^{-1}\left(\langle\nabla\rangle_{m}\left(v\langle\nabla\rangle_{m} w\right)-v\langle\nabla\rangle_{m}^{2} w\right)\right\|_{H^{1}} \lesssim\left\|\langle\nabla\rangle_{m}\left(v\langle\nabla\rangle_{m} w\right)-v\langle\nabla\rangle_{m}^{2} w\right\|_{L^{2}} \\
& \lesssim\|\nabla v\|_{L^{4}}\left\|\langle\nabla\rangle_{m} w\right\|_{L^{4}} \lesssim\|v\|_{H^{\frac{7}{4}}}\|w\|_{H^{\frac{7}{4}}} .
\end{aligned}
$$


We thus obtain that

Corollary 26. For for every $u_{0} \in H^{\frac{7}{4}}\left(\mathbb{T}^{3}\right), u_{1} \in H^{\frac{3}{4}}\left(\mathbb{T}^{3}\right)$ Let $T>0$ and $z \in \mathcal{C}\left([0, T], H^{\frac{7}{4}}\left(\mathbb{T}^{3}\right)\right) \cap$ $\mathcal{C}^{1}\left([0, T], H^{\frac{3}{4}}\left(\mathbb{T}^{3}\right)\right)$ the unique solution of $(90)$, with $g(z)=z^{2}$. Let $u^{n}$ denote the numerical solution given by (6). Then there exists $C_{T}>0$ such that

$$
\left\|z(n \tau)-\operatorname{Re} u^{n}\right\|_{H^{1}}+\left\|\partial_{t} z(n \tau)-\operatorname{Im} u^{n}\right\|_{L^{2}} \leq C_{T} \tau^{2}, \quad 0 \leq n \tau \leq T .
$$

Note that second-order convergence of classical schemes for the Klein-Gordon equation (90) requires (at least) $H^{2}$ solutions. In a similar way we can treat the wave equation

$$
\partial_{t t} z-\Delta z=h(z)
$$

by setting $g(z)=h(z)+m^{2} z$ for some $m \neq 0$ such that $\partial_{t t} z-\Delta z+m^{2} z=g(z)$.

\section{Technical Lemma}

In this section, we shall use the Littlewood-Paley decomposition on $\mathbb{T}^{d}$. Let us recall some basic facts, we refer to the book [1] for example for the proofs. We take a partition of unity of the form

$$
1=\phi_{-1}(\xi)+\sum_{k \geq 0} \phi_{k}(\xi)
$$

where $\phi_{-1}$ is supported in the ball $\bar{B}(0,1)$ and each $\phi_{k}(\xi)=\phi\left(\xi / 2^{k}\right), k \geq 0$ is supported in the annulus $2^{k-1} \lesssim|\xi| \lesssim 2^{k+1}$. We can then decompose any tempered distribution as

$$
u=\sum_{k \geq-1} \Delta_{k} u, \quad \mathcal{F}\left(\Delta_{k} u\right)(\xi)=\phi_{k}(\xi) \hat{u}(\xi)
$$

note that we can use the Fourier transform on $\mathbb{R}^{d}$ or on $\mathbb{T}^{d}$, in this case $\xi \in \mathbb{Z}^{d}$. We shall use the following facts (Bernstein inequality): For every $\sigma \geq 0$ and every $p \in[1, \infty]$, there exist constants $c>0$ and $C>0$ such that for every $k \geq 0$, we have

$$
c 2^{\sigma k}\left\|\left(\phi_{k}(-i \nabla)\right) u\right\|_{L^{p}} \leq\left\||-i \nabla|^{\sigma}\left(\phi_{k}(-i \nabla) u\right)\right\|_{L^{p}} \leq C 2^{\sigma k}\left\|\left(\phi_{k}(-i \nabla) u\right)\right\|_{L^{p}} .
$$

For two functions $u, v$, we have Bony's decomposition

$$
u v=T_{u} v+T_{v} u+R(u, v)
$$

where we set

$$
T_{a} b=\sum_{q} S_{q-1} a \Delta_{q} b, \quad S_{q-1} a=\sum_{k \leq q-2} \Delta_{k} a, \quad R(u, v)=\sum_{|p-q| \leq 2} \Delta_{p} a \Delta_{q} b .
$$

We shall first prove

Lemma 27. For $u, v \in H^{\frac{1}{2}+\frac{d}{4}}$, we have the estimate

$$
\||\nabla|(u v)-|\nabla| u v-u|\nabla| v\|_{L^{2}} \lesssim\|u\|_{H^{\frac{1}{2}+\frac{d}{4}}}\|v\|_{H^{\frac{1}{2}+\frac{d}{4}}} \cdot
$$

This estimate also holds with $|\nabla|$ replaced by $\langle\nabla\rangle_{m}$.

Proof. The fact that the same estimate holds for $|\nabla|$ and $\langle\nabla\rangle_{m}$ just follows from the observation that $\langle\nabla\rangle_{m}-|\nabla|$ is a bounded operator. We shall thus prove (103) We can use the decomposition (102). The result then follows if we can estimate the following terms

$$
I=|\nabla|\left(T_{u} v\right)-T_{|\nabla| u} v-T_{u}|\nabla| v, \quad I I=R(u,|\nabla| v), I I I=|\nabla| R(u, v)
$$

and symmetric terms.

By using Theorem 2.85 of [1], we get that

$$
\|I I\|_{L^{2}}+\|I I I\|_{L^{2}} \lesssim\|u\|_{H^{\frac{1}{2}+\frac{d}{4}}}\|v\|_{H^{\frac{1}{2}+\frac{d}{4}}}
$$


so that we only need to estimate $I$. By using the frequency localization, we get that

$$
\|I\|_{L^{2}}^{2} \lesssim \sum_{q}\left\||\nabla|\left(S_{q-1} u \Delta_{q} v\right)-|\nabla| S_{q-1} u \Delta_{q} v-S_{q-1} u|\nabla| \Delta_{q} v\right\|_{L^{2}}^{2} .
$$

By using the fourier Transform we need to estimate

$$
I_{q}=\sum_{k}\left|\sum_{l} m(k-l, l) \widehat{\Delta_{q} v}(k-l) \widehat{S_{q-1} u}(l)\right|^{2}
$$

where $|m(\xi, \eta)|=|| \xi+\eta|-| \xi|-| \eta|| \lesssim|\xi||\eta| /(|\xi+\eta|+|\xi|+|\eta|)$. We remind that due to the frequency localisation in the Littlewood-Paley decomposition, we have $|l| \lesssim 2^{q-1}$ and $2^{q-1} \leq|k-l| \leq 2^{q+1}$. By using Cauchy-Schwarz and Fubini, we then get that

$$
I_{q} \lesssim \sup _{2^{q-1} \leq|\xi| \leq 2^{q+1}} \sum_{|l| \leq 2^{q-2}} \frac{|m(\xi, l)|^{2}}{1+|l|^{1+\frac{d}{2}}}\|u\|_{H^{\frac{1}{2}+\frac{d}{4}}}^{2}\left\|\Delta_{q} v\right\|_{L^{2}}^{2} .
$$

Since $|m(\xi, l)| \leq|l|$, we have that

$$
\sum_{|l| \leq 2^{q-2}} \frac{|m(\xi, l)|^{2}}{1+|l|^{1+\frac{d}{2}}} \lesssim 2^{q\left(1+\frac{d}{2}\right)}
$$

Therefore, we get that

$$
\|I\|_{L^{2}}^{2} \lesssim \sum_{q} I_{q} \lesssim 2^{q\left(1+\frac{d}{2}\right)}\|u\|_{H^{\frac{1}{2}+\frac{d}{4}}}^{2}\left\|\Delta_{q} v\right\|_{L^{2}}^{2} \lesssim\|u\|_{H^{\frac{1}{2}+\frac{d}{4}}}^{2}\|v\|_{H^{\frac{1}{2}+\frac{d}{4}}}^{2}
$$

where the last inequality comes from the Bernstein inequality and the almost orthogonality of the terms. This concludes the proof.

Lemma 28. For $d=1, u \in H^{\frac{3}{4}}(\mathbb{T})$, we have

$$
\left\|\langle\nabla\rangle_{m} \sin u-\cos u\langle\nabla\rangle_{m} u\right\|_{L^{2}} \lesssim C\left(\|u\|_{H^{\frac{3}{4}}}\right) .
$$

Proof. By using for example [21] Theorem 5.2.5 (still valid on the torus), we have that for $u \in H^{s}$, $s>d / 2$ (we take $s=3 / 4$ ), $\sin u-T_{\cos u} u \in H^{2 s-\frac{d}{2}}$. We thus get that

$$
\langle\nabla\rangle_{m} \sin u=\langle\nabla\rangle_{m}\left(T_{\cos u} u\right)+\mathcal{R}_{1}
$$

with $\mathcal{R}_{1} \in H^{2 s-\frac{3}{2}}$. Next, we can write that

$$
\cos u\langle\nabla\rangle_{m} u=T_{\cos u}\langle\nabla\rangle_{m} u+T_{\langle\nabla\rangle_{m} u} \cos u+R\left(\cos u,\langle\nabla\rangle_{m} u\right) .
$$

By using again Theorem 2.85 of [1], we have that

$$
\left\|R\left(\cos u,\langle\nabla\rangle_{m} u\right)\right\|_{L^{2}} \lesssim\|\cos u\|_{H^{\frac{3}{4}}}\left\|\langle\nabla\rangle_{m} u\right\|_{H^{-\frac{1}{4}}} \lesssim C\left(\|u\|_{H^{\frac{3}{4}}}\right) .
$$

By using also Theorem 2.82 of [1], we also have

$$
\left\|T_{\langle\nabla\rangle_{m} u} \cos u\right\|_{L^{2}} \lesssim\left\|\langle\nabla\rangle_{m}^{\frac{1}{4}} u\right\|_{L^{\infty}}\|\cos u\|_{H^{\frac{3}{4}}} \lesssim C\left(\|u\|_{H^{\frac{3}{4}}}\right) .
$$

Therefore, it only remains to estimate

$$
\langle\nabla\rangle_{m}\left(T_{\cos u} u\right)-T_{\cos u}\langle\nabla\rangle_{m} u .
$$

By frequency localization, we have that

$$
\left\|\langle\nabla\rangle_{m}\left(T_{\cos u} u\right)-T_{\cos u}\langle\nabla\rangle_{m} u\right\|_{L^{2}}^{2} \lesssim \sum_{k}\left\|\langle\nabla\rangle_{m}\left(S_{k-1} \cos u \Delta_{k} u\right)-S_{k-1} \cos u\langle\nabla\rangle_{m} \Delta_{k} u\right\|_{L^{2}}^{2},
$$


therefore, by using Lemma 27, we get that

$$
\begin{aligned}
\left\|\langle\nabla\rangle_{m}\left(T_{\cos u} u\right)-T_{\cos u}\langle\nabla\rangle_{m} u\right\|_{L^{2}}^{2} \lesssim \sum_{k}\left\|S_{k-1} \cos u\right\|_{H^{\frac{3}{4}}}^{2}\left\|\Delta_{k} u\right\|_{H^{\frac{3}{4}}}^{2} & \\
& \lesssim\|\cos u\|_{H^{\frac{3}{4}}}^{2} \sum_{k}\left\|\Delta_{k} u\right\|_{H^{\frac{3}{4}}}^{2} \lesssim\|\cos u\|_{H^{\frac{3}{4}}}^{2}\|u\|_{H^{\frac{3}{4}}}^{2} .
\end{aligned}
$$

We conclude by using again that

$$
\|\cos u\|_{H^{\frac{3}{4}}} \lesssim 1+\|u\|_{H^{\frac{3}{4}}}
$$

This concludes the proof.

\section{ACKNowledgement}

This project has received funding from the European Research Council (ERC) under the European Unions Horizon 2020 research and innovation programme (grant agreement No. 850941).

\section{REFERENCES}

[1] H. Bahouri, J.-Y. Chemin, R. Danchin. Fourier Analysis and Nonlinear Partial Differential Equations. Springer, Heidelberg, 2011

[2] S. Baumstark, K. Schratz, Uniformly accurate oscillatory integrators for the Klein-Gordon-Zakharov system from low- to high-plasma frequency regimes. SIAM J. Numer. Anal. 57:429-457 (2019)

[3] S. Baumstark, E. Faou, K. Schratz, Uniformly accurate oscillatory integrators for Klein-Gordon equations with asymptotic convergence to the classical NLS splitting. Math. Comp. 87:1227-1254 (2018)

[4] C. Besse, B. Bidégaray, S. Descombes, Order estimates in time of splitting methods for the nonlinear Schrödinger equation, SIAM J. Numer. Anal. 40:26-40 (2002).

[5] Y. Bruned, K. Schratz, Resonance based schemes for dispersive equations via decorated trees (preprint) http://arxiv.org/abs/2005.01649

[6] N. Burq, P. Gérard, N. Tzvetkov, Strichartz inequalities and the nonlinear Schrödinger equation on compact manifolds. Amer. J. Math. 126:569-605 (2004)

[7] E. Celledoni, D. Cohen, B. Owren, Symmetric exponential integrators with an application to the cubic Schrödinger equation. Found. Comput. Math. 8:303-317 (2008).

[8] E. Faou, Geometric Numerical Integration and Schrödinger Equations. European Math. Soc. Publishing House, Zürich 2012.

[9] E. Hairer, S. P. Nørsett, G. Wanner, Solving Ordinary Differential Equations I. Nonstiff Problems. Second edition. Springer, Berlin 1993.

[10] E. Hairer, C. Lubich, G. Wanner, Geometric Numerical Integration. Structure-Preserving Algorithms for Ordinary Differential Equations. Second edition, Springer, Berlin 2006.

[11] M. Hochbruck, A. Ostermann, Exponential integrators. Acta Numer. 19:209-286 (2010).

[12] M. Hochbruck, J. Leipold, A. Ostermann, On the convergence of Lawson methods for semilinear stiff problems. Numer. Math. 145:553-580 (2020)

[13] H. Holden, K. H. Karlsen, K.-A. Lie, N. H. Risebro, Splitting for Partial Differential Equations with Rough Solutions. European Math. Soc. Publishing House, Zürich 2010.

[14] M. Hofmanová, K. Schratz, An oscillatory integrator for the KdV equation, Numer. Math. 136:1117-1137 (2017).

[15] M. Knöller, A. Ostermann, K. Schratz, A Fourier integrator for the cubic nonlinear Schrödinger equation with rough initial data. SIAM J. Numer. Anal. 57:1967-1986 (2019).

[16] B. Leimkuhler, S. Reich, Simulating Hamiltonian dynamics. Cambridge Monographs on Applied and Computational Mathematics 14. Cambridge University Press, Cambridge, 2004

[17] J. D. Lawson, Generalized Runge-Kutta processes for stable systems with large Lipschitz constants. SIAM J. Numer. Anal. 4:372-380 (1967).

[18] Dong Li, On Kato-Ponce and fractional Leibniz. Rev. Mat. Iberoam. 35 (2019), no. 1, 23-100.

[19] C. Lubich, On splitting methods for Schrödinger-Poisson and cubic nonlinear Schrödinger equations. Math. Comp. 77:2141-2153 (2008).

[20] R.I. McLachlan, G.R.W. Quispel, Splitting methods, Acta Numer. 11:341-434 (2002).

[21] G. Métivier, Para-differential calculus and applications to the Cauchy problem for nonlinear systems. Centro di Ricerca Matematica Ennio De Giorgi (CRM) Series, 5. Edizioni della Normale, Pisa, 2008. 
[22] A. Ostermann, K. Schratz, Low regularity exponential-type integrators for semilinear Schrödinger equations, Found. Comput. Math. 18:731-755 (2018).

[23] A. Ostermann, F. Rousset, K. Schratz, Error estimates of a Fourier integrator for the cubic Schrödinger equation at low regularity (to appear in Found. Comput. Math.) https://arxiv.org/abs/1902.06779.

[24] A. Ostermann, F. Rousset, K. Schratz, Fourier integrator for periodic NLS: low regularity estimates via discrete Bourgain spaces https://arxiv.org/abs/2006.12785.

[25] A. Ostermann, C. Su, Two exponential-type integrators for the "good" Boussinesq equation. Numer. Math. 143:683-712 (2019)

[26] J.M. Sanz-Serna, M.P. Calvo, Numerical Hamiltonian Problems. Chapman and Hall, London, 1994

[27] K. Schratz, Y. Wang, X. Zhao, Low-regularity integrators for nonlinear Dirac equations. (to appear in Math. Comput.) https://arxiv.org/abs/1906.09413

[28] Y. Wu, X. Zhao, Optimal convergence of a second order low-regularity integrator for the KdV equation. preprint 2019 https://arxiv.org/abs/1910.07367

Laboratoire de Mathématiques d’Orsay (UMR 8628), Université Paris-Saclay, 91405 Orsay Cedex, France (F. Rousset)

Email address: frederic.rousset@universite-paris-saclay.fr

LJLL (UMR 7598), Sorbonne Université, UPMC, 4 place Jussieu, 75005, Paris, France (K. Schratz)

Email address: katharina.schratz@ljll.math.upmc.fr 